\title{
Full-Chip Routing Considering Double-Via Insertion
}

\author{
Huang-Yu Chen, Student Member, IEEE, Mei-Fang Chiang, Student Member, IEEE, \\ Yao-Wen Chang, Member, IEEE, Lumdo Chen, and Brian Han
}

\begin{abstract}
As the technology node advances into the nanometer era, via-open defects are one of the dominant failures due to the copper cladding process. To improve via yield and reliability, redundant-via insertion is a highly recommended technique proposed by foundries. Traditionally, double-via insertion is performed at the postlayout stage. The increasing design complexity, however, leaves very limited space for postlayout optimization. It is thus desirable to consider the double-via insertion at both the routing and postrouting stages. In this paper, we present a new full-chip gridless routing system considering double-via insertion for yield enhancement. To fully consider double vias, the router applies a novel two-pass, bottom-up routability-driven routing framework and features a new redundant-via aware detailed maze routing algorithm (which could be applied to both gridless and grid-based routing). We also propose a graph-matching based post-layout double-via insertion algorithm to achieve a higher insertion rate. In particular, the algorithm is optimal for grid-based routing with up to three routing layers and the stacked-via structure. Experiments show that our methods significantly improve the via count, number of dead vias, double-via insertion rates, and running times.
\end{abstract}

Index Terms-Design for manufacturability, detailed routing, double via, global routing, gridless routing, physical design, redundant via.

\section{INTRODUCTION}

A $S$ integrated circuit process geometries shrink to $65 \mathrm{~nm}$ and below, yield and reliability become firstorder cost metrics. Via-open defects are one of the important failures. A via may fail due to various reasons such as random defects, electromigration, cut misalignment, and/or thermalstress-induced voiding effects. Via failures significantly reduce the manufacturing yield and chip performance.

Manuscript received February 24, 2007; revised July 21, 2007. This work was supported in part by the National Science Council of Taiwan under Grant NSC 96-2752-E-002-008-PAE, Grant NSC 96-2628-E-002-248-MY3, Grant NSC 96-2628-E-002-249-MY3, and Grant NSC 96-2221-E-002-245. This paper was presented in part at the 43rd Design Automation Conference, July 24-28, 2006, San Francisco, CA. This paper was recommended by Associate Editor L. Scheffer.

H.-Y. Chen is with the Graduate Institute of Electronics Engineering, National Taiwan University, Taipei 106, Taiwan, R.O.C. (e-mail: yellowfish@eda. ee.ntu.edu.tw).

M.-F. Chiang is with the Graduate School of Information, Production and Systems, Waseda University, Kitakyushu 808-0135, Japan (e-mail: annika@ ruri.waseda.jp).

Y.-W. Chang is with the Graduate Institute of Electronics Engineering and Department of Electrical Engineering, National Taiwan University, Taipei 106, Taiwan, R.O.C. (e-mail: ywchang@cc.ee.ntu.edu.tw).

L. Chen and B. Han are with United Microelectronics Corporation, Hsinchu 300, Taiwan, R.O.C. (e-mail: lumdo_chen@umc.com; soe_han@ yahoo.com)

Digital Object Identifier 10.1109/TCAD.2008.917597

\section{A. Redundant-Via Insertion}

To improve via yield and reliability, redundant-via insertion is a highly recommended technique proposed by foundries. If one via fails, a redundant via can serve as a fault-tolerant substitute for the failing one. As reported in [24], double vias lead to $10 \times-100 \times$ smaller failure rates than single vias. Existing approaches are often for postlayout optimization by replacing a single via with a double-via structure as long as it does not create any design-rule violations [3], [18]. The increasing design complexity, however, leaves very limited space for postlayout optimization. It has been reported that inserting redundant vias during routing can improve the double-via insertion rates by $15 \%-25 \%$ than the postlayout optimization, at the cost of routability degradation. As a result, major foundry, such as the Taiwan Semiconductor Manufacturing Company, has recommended performing double-via insertion during routing and postlayout optimization as a defect-tolerant process for 90- and 65-nm manufacturing processes [22]. Therefore, it is desired to consider the double-via insertion at both the routing and postrouting stages for better tradeoff in routability and double-via insertion rates.

Recently, Xu et al. [26] proposed pioneering work to consider double-via insertion during maze routing. By assigning double-via costs to the routing graph, they formulated the problem as a multiobjective maze routing problem and applied Lagrangian relaxation to solve it. However, they only consider the redundant via at the detailed routing stage, and the high time complexity of Lagrangian relaxation limits the feasible problem size to be within hundreds of nets. Yao et al. [27] developed a grid-based router, which features via-minimization global routing, followed by double-via aware detailed routing; the work claimed that the postlayout double-via insertion (PDVI) problem can be solved by a maximum bipartite matching formulation, which was recently shown to be incorrect for some cases in [18]. Lee and Wang [18] instead formulated the double-via insertion problem as a maximum independent set (MIS) problem. Since the MIS problem for a general graph is nondeterministic polynomial time (NP)-complete [15], they resorted to heuristics to handle the problem.

\section{B. Routing Framework Evolution}

The continuously increasing design complexity imposes severe challenges for modern routers. Nowadays, a modern chip may contain several billion transistors and has over one million nets. Consequently, the routing frameworks are evolving from the flat framework to the hierarchical and multilevel frameworks. Many routing algorithms adopt a two-stage flat 


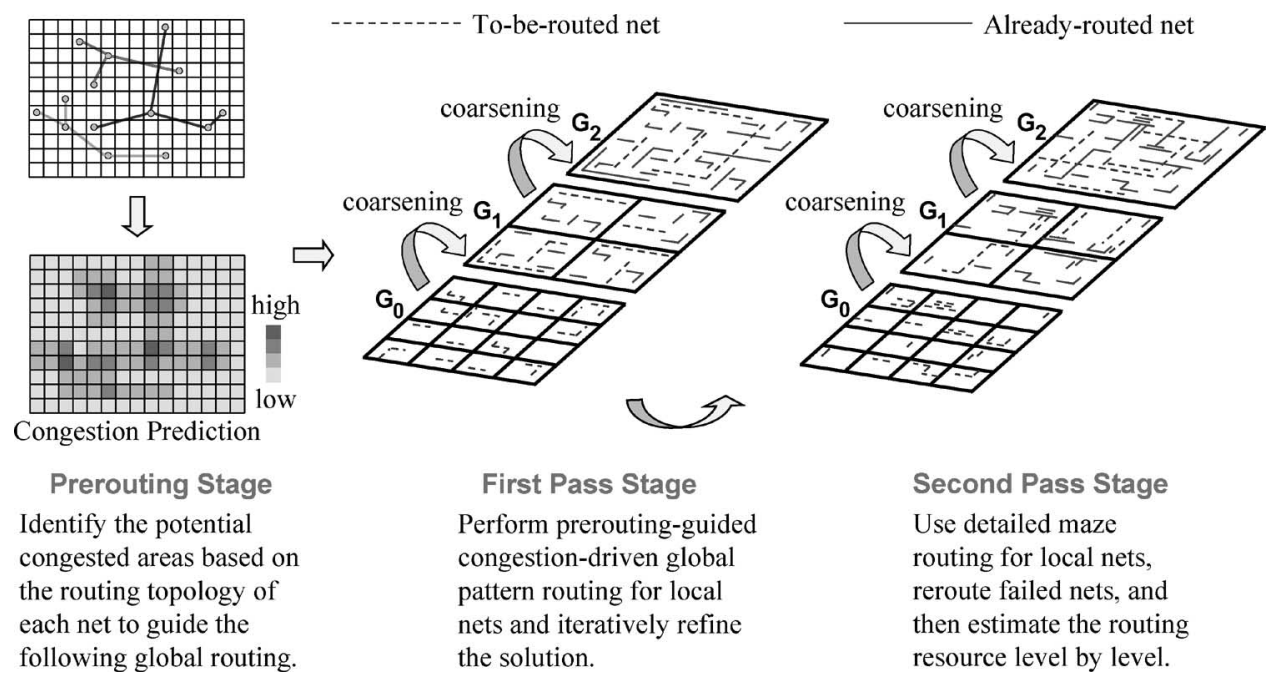

Fig. 1. New two-pass bottom-up routing framework.

framework of global routing, followed by detailed routing [2], [17]. Global routing first partitions the routing region into tiles and then decides tile-to-tile paths for all nets, whereas detailed routing determines exact tracks and vias for nets within each tile.

However, the flat framework does not scale well as the design size increases. To cope with the scalability problem, hierarchical and multilevel frameworks are proposed. The hierarchical framework uses the divide-and-conquer approach to handle smaller subproblems independently [12], [19], [21]. Although the hierarchical approach can scale to larger designs, it has a drawback of lacking interactions among routing subregions and thus limits the solution quality. To remedy the deficiencies, researchers have proposed various multilevel frameworks to handle large-scale routing problems. The traditional $\Lambda$-shaped multilevel routing framework consists of bottom-up coarsening, followed by top-down uncoarsening (e.g., MARS [10], [11], MR [6], CMR [13], and MGR [8]), whereas the V-shaped one consists of top-down uncoarsening, followed by bottomup coarsening (e.g., VMGR [9]). The coarsening stage is a bottom-up approach that iteratively groups a set of circuit components (e.g., routing tiles) based on a predefined cost metric. In contrast, the uncoarsening stage iteratively ungroups a set of previously clustered circuit components in a top-down manner. The multilevel frameworks demonstrate the superior capability of handling large-scale routing problems and the versatility of tackling modern nanometer electrical effects, such as crosstalk [13] and optical proximity correction [8]. It is also observed that the $\Lambda$-shaped multilevel framework can handle local circuit effects (such as routability, congestion, and via minimization) better since it works in a bottom-up manner and deals with local routing regions first (i.e., route shorter local nets and then longer global nets) [6], [16]. In contrast, the V-shaped multilevel framework is more suitable for handling global electrical effects (such as crosstalk and critical-path delay) since it works in a top-down manner and copes with global routing regions first [9].

\section{Our Contributions}

To maximize the redundant-via insertion rate, we consider redundant-via insertion during routing as well as postlayout optimization. In this paper, we present a new full-chip gridless routing system called Two-pass Bottom-up gridless Router (TBR), considering redundant-via insertion for yield enhancement. To fully consider redundant vias, the router is based on a novel two-pass bottom-up routability-driven routing framework. Different from the previous routing frameworks, TBR adopts a three-stage technique of a prerouting stage, followed by a bottom-up global routing stage and then followed by a bottom-up detailed routing stage. Fig. 1 illustrates an the new framework. The motivation for the two-pass bottom-up approach lies in the observation that it is more effective to route shorter local nets first for routability and via optimization. Although the $\Lambda$-shaped multilevel framework also performs bottom-up coarsening first, it refines the solution top-down during the following uncoarsening stage. By using the two-pass bottom-up approach, we can take full advantage of processing local nets first at the two routing passes for routability and via optimization.

For congestion minimization, TBR starts with a prerouting stage, which estimates the potential congested areas based on the routing topology of each net. Guided by this estimation, the following routing would favor the paths that minimize congestions. Then, the first bottom-up global routing pass is performed; it starts from coarsening the finest level to the coarsest level. At each level, the L- or Z-shaped pattern routing [16] is used for congestion-driven global routing. When an initial global routing solution is generated, net-ordering dependence is explored, and iterative refinement is performed for congestion optimization. It will be clear that the L- and Z-shaped global pattern routing leads to the via count reduction in the following detailed routing stage. The second bottomup routing pass is for detailed routing. As in the first pass, it proceeds from coarsening the finest level to the coarsest level. At each level, redundant-via aware detailed maze routing (could be gridless or grid-based routing) is performed, and ripup/reroute procedures are applied for failed nets. By performing the redundant-via aware detailed routing, we can minimize the number of vias that do not have any feasible redundant-via candidates and reserve more adjacent space for each via to facilitate the PDVI and thus increase the final redundant-via insertion rate. 


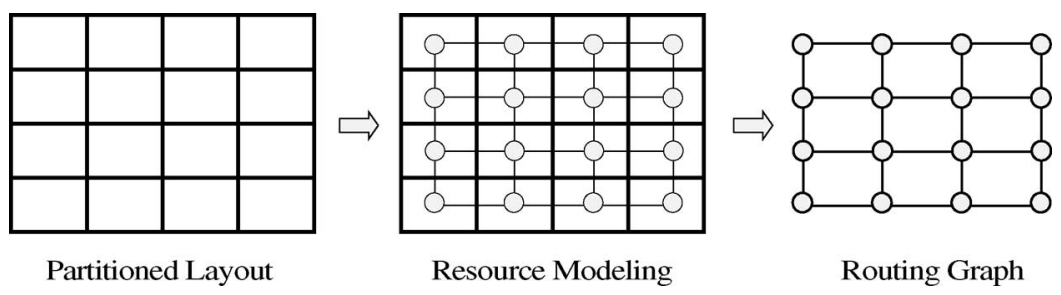

Fig. 2. Routing graph.

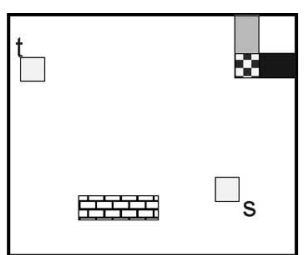

(a)

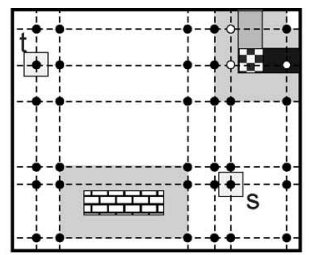

(b)

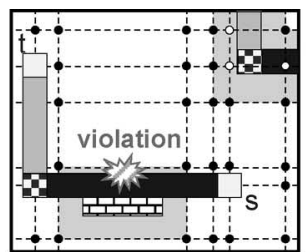

(c)

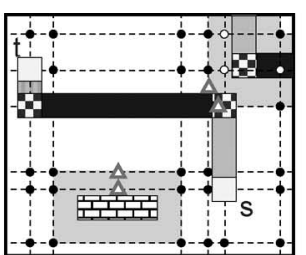

(d)

Metal $1 \square$ Metal $2 \square$ Pin $\mathbf{D}$ Via Obstacle $\bullet$ Routable Node ${ }^{\circ}$ Unroutable Node $\Delta$ Additional Check

Fig. 3. Gridless detailed routing model. (a) Detailed routing instance. (b) Implicit connection graph. (c) Infeasible detailed routing path. (d) Design-rule-correct detailed routing path.

We also propose a graph-matching based post-layout doublevia insertion algorithm to achieve a higher insertion rate. In particular, the algorithm is optimal for grid-based routing with up to three routing layers and the stacked-via structure. With the optimal algorithm for the restricted problem, we then extend it to handle the general case of any routing layer and via structure.

Experimental results show that our routing system reduces the via count by $1.20 \times$ compared with the state-of-the-art gridless router [8]-[10], and our redundant-via aware detailed router can effectively obtain fewer dead vias by $1.44 \times$. Compared with the state-of-the-art double-via insertion algorithm [18] under the stacked-via formulation, our post-layout doublevia insertion algorithm can achieve $98.6 \%$ double-via insertion rate with at least $70.8 \times$ runtime speedup.

The rest of this paper is organized as follows: Section II describes the routing model and the postlayout redundantvia insertion problem. Section III presents our novel two-pass bottom-up routing framework considering redundant-via insertion. Section IV presents our PDVI algorithm. Experimental results are reported in Section $\mathrm{V}$, and conclusions are given in Section VI.

\section{PRELIMINARIES}

In this section, we give the routing models, the two-pass bottom-up routing framework, and the formulation for the PDVI problem.

\section{A. Global Routing Model}

Our routing algorithm is based on a graph-search technique guided by the congestion information associated with routing regions and net topologies. The router assigns higher costs to nets passing through congested areas to balance the net distribution among routing regions.

Before we can apply the graph-search technique to routing, we first need to model the routing resource as a routing graph whose topology can represent the chip structure. Fig. 2 illustrates the graph modeling. For the modeling, we first partition a chip into an array of rectangular global cells (GCs), each of which may accommodate tens of routing tracks in each dimension. A node in the routing graph represents a GC in the chip, whereas an edge denotes the boundary between two adjacent GCs. Each edge is assigned a capacity according to the physical area or the size of a GC. A global router finds GCto-GC paths for all nets to guide the detailed router. The goal of global routing is to route as many nets as possible without violating any capacity constraint of each edge and to meet any other specified optimization constraints.

\section{B. Detailed Gridless Routing Model}

The goal of detailed routing is to find a design-rule-correct path for each connection while meeting every specified constraint. Our gridless detailed routing applies a graph-search technique based on an implicit connection graph used in [10], with a modification to guarantee the correctness of the searched path. Fig. 3(a) gives a detailed routing instance with source $s$ and target $t$, and Fig. 3(b) shows the corresponding implicit connection graph constructed based on $s, t$, and the obstacle zones. An obstacle zone is a minimum expansion of an existing obstacle (e.g., an already-routed wire) such that any new routing wire lying on the boundaries of this obstacle zone would not violate any design rule. A node in the implicit connection graph is an unroutable node if it is inside an obstacle zone; it is a routable node otherwise. The black and white circles in Fig. 3(b) represent the routable and unroutable nodes, respectively.

It should be noted that, if there exists a path formed by two successive routable nodes belonging to the same obstacle zone, it might be considered a feasible route in [10]. As shown in Fig. 3(c), nevertheless, a path might illegally cut across one obstacle zone through two routable nodes lying on the boundaries of this obstacle zone. To guarantee the correctness of the searched path, Chen et al. [8], [9] added horizontal and vertical middle lines for each obstacle zone. However, this approach would significantly increase the search space and, 
thus, the running time. To remedy this deficiency, we search the path by an additional check to see if the midpoint of the two routable nodes belonging to the same obstacle zone is an interior point of this obstacle zone. Fig. 3(d) shows the triangular points representing these additional checks. Note that each check operation takes only constant time. In this way, a design-rule-correct detailed routing path can be efficiently guaranteed.

\section{Two-Pass Bottom-Up Routing Framework Model}

As illustrated in Fig. 1, $G_{k}$ corresponds to the routing graph of level $k$. The first bottom-up routing pass is the global routing stage, which starts from coarsening the finest level (level 0) to the coarsest level. At each level $k$, our global router finds routing paths for the local connections (those connections that entirely sit inside $\mathrm{GC}_{k+1}$, where $\mathrm{GC}_{k}$ is the $\mathrm{GC}$ of level $k$ ). After global routing of level $k$ is performed, we merge four $\mathrm{GC}_{k} \mathrm{~s}$ into a larger $\mathrm{GC}_{k+1}$ and, at the same time, perform resource estimation for use at the next level $k+1$. Coarsening continues until the number of GCs at a level is below a threshold.

The second bottom-up routing pass is the detailed routing stage. As the first pass, it processes from coarsening the finest level to the coarsest level. At each level, a detailed maze routing is performed, and rip-up/reroute procedures are applied for failed nets. The process continues until we reach the coarsest level when the final routing solution is obtained.

\section{PDVI}

After the detailed routing, we have a resulting layout with various types of vias. For a via, a redundant-via candidate is a candidate position where a redundant via can be inserted for this via without violating any design rule. Note that a redundant via is typically horizontally or vertically inserted at a fixed distance from a via for practical applications; thus, we can treat a via and its redundant via as a pair. Therefore, we refer to a redundantvia candidate as one of the four positions with a fixed distance (specified by the design rules) from a via in the left, right, top, and bottom directions, same as most existing works [3], [18]. A via is an alive via if it has at least one redundant-via candidate for insertion; otherwise, it is a dead via. A critical via refers to an alive via with exactly one redundant-via candidate. A via is either a single (conventional) via or a stacked via. A stacked via is the via consisting of at least two vertically stacked single vias.

We treat both the stacked via and the single via as one unit via since, from the connection viewpoint, if any one single via contained in a stacked via is not paired with a redundant via, this stacked via is still not protected. The formulation of the postlayout redundant-via insertion problem is defined as follows.

- Problem PDVI: Given a postrouting layout, pair each alive via with a redundant via as many as possible such that no design rule is violated after the double-via insertion.

\section{Redundant-Via Aware Routing}

For via yield enhancement during routing, we shall try to: 1) minimize the via count to reduce the failure probability and
2) plan the double-via positions for each via to ensure that a double via can be inserted wherever needed in the later PDVI process.

To deal with the simultaneous optimization, we propose a novel two-pass bottom-up routing framework that adopts a three-stage technique of a prerouting stage, followed by a bottom-up global routing stage and a bottom-up detailed routing stage (see Fig. 1). The prerouting stage identifies the potentially congested areas to guide the following routing for congestion optimization. The two bottom-up routing passes tend to route shorter nets first level by level, which directly contributes to the routability enhancements and viacount minimization. Based on this routability- and via-aware framework, we develop a two-pass bottom-up full-chip gridless routing system named TBR. Specifically, the TBR consists of the following: 1) a congestion-driven prerouting stage; 2) a via-minimization global routing stage; and 3) a redundantvia planning detailed routing stage. We detail the three stages in the following sections.

\section{A. Congestion-Driven Prerouting Stage}

To improve routability, the works [6], [8], and [9] integrated global routing, detailed routing, and resource estimation together at each level of the framework, leading to more accurate routing resource estimation. However, this approach might confine the optimization freedom since global routing and detailed routing are intertwined with each other.

In order to consider more objectives for congestion minimization, the TBR features a prerouting stage that identifies the potentially congested areas based on the routing topology of each net. With the prerouting, the TBR can separately perform global routing and detailed routing and leave more flexibility in dealing with the redundant-via-related objectives.

Given a netlist, we first construct a minimum spanning tree (MST) for each net and then decompose each net into two-pin connections, with each connection corresponding to an edge of the MST. The MST topology leads to the minimum total wire length; thus, congestion is often easier to be controlled than other topologies. The TBR then preestimates the congestion in the routing graph for all two-pin connections using the probabilistic congestion model, which has recently been successfully applied to placement [4], floor planning [14], and routing [20], [25], and is generally believed to have the ability to alleviate the net-ordering problem in sequential routing. The TBR preevaluates the congestion as the average number of global one-bend and two-bend routes that might pass through the boundary of adjacent GCs. For a two-pin connection $c$, we first explore all possible one- and two-bend global routes from its source $s$ to its target $t$, which are denoted by set $P_{c}$. All routes in $P_{c}$ are the candidates of global routing solutions for $c$. For a boundary $b_{i}$ between two GCs, let $N_{c}(i)=\{r \in$ $P_{c} \mid r$ is the route passing through $\left.b_{i}\right\}$; then, the estimated congestion of $b_{i}$ with respect to $c$ is equal to $\left|N_{c}(i)\right| /\left|P_{c}\right|$. For example, as shown in Fig. 4(a), connection $c$ has five possible one- and two-bend routes from source $s$ to target $t$. Fig. 4(b) gives the number of routes passing through each GC boundary $b_{i},\left|N_{c}(i)\right|$, and Fig. 4(c) shows the congestion estimation of $c$ in the routing graph. 


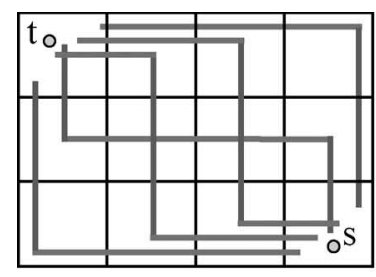

(a)

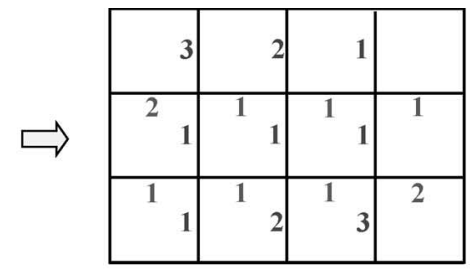

(b)

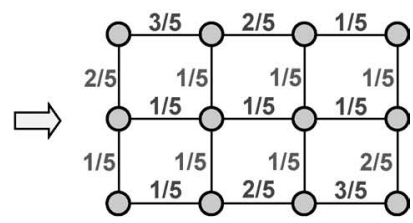

(c)

Fig. 4. Probabilistic congestion estimation. (a) Two one-bend and three two-bend routes from $s$ to $t$. (b) Number of routes through each boundary. (c) Preestimation congestion in the routing graph.

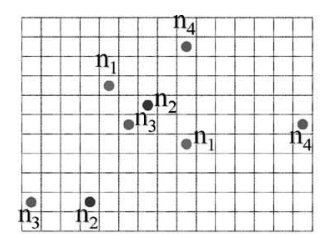

(a)

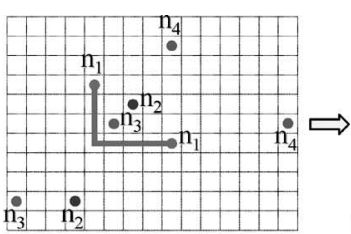

$n_{3} \quad n_{2}$
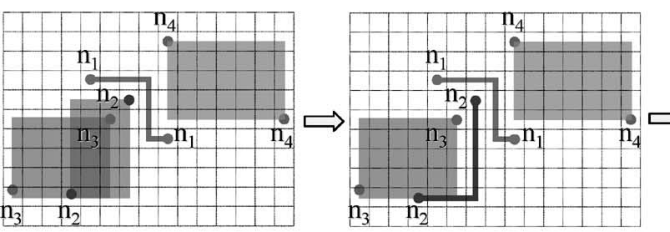

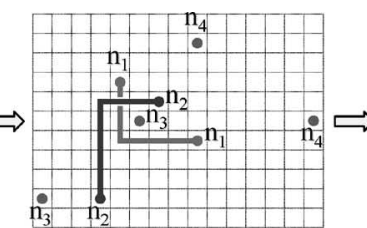

(b)
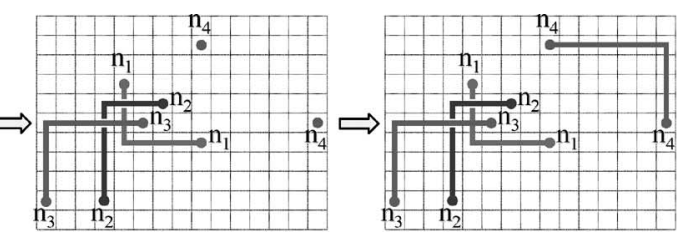

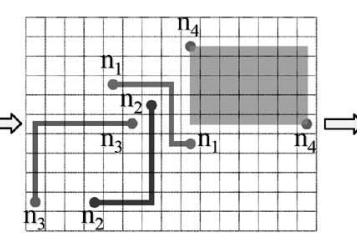

(c)
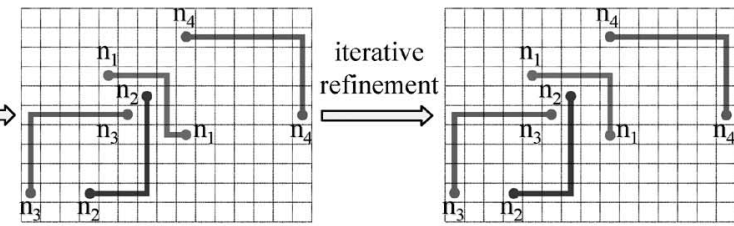

Fig. 5. Effect of congestion estimation. (a) Global-routing instance. (b) Routing result generated by a traditional net-ordered global router. (c) Better routing result generated by the prerouting-guided congestion-driven global router, followed by iterative refinement.

\section{B. Via-Minimization Global Routing Stage}

The first bottom-up global-routing pass is a coarsening process starting from the finest level to the coarsest level. Our global routing is based on the approach used for pattern routing [16]. Let the routing graph of level 0 be $G_{0}=\left(V_{0}, E_{0}\right)$ and the global routing result for a local connection $c$ be $R_{c}=$ $\left\{e \in E_{0} \mid e\right.$ is the edge chosen for routing $\}$. For the congestion control, we define the cost function of the global routing result $R_{c}$ as follows:

$$
\Psi_{R_{c}}=\alpha \max _{e \in R_{c}}\left(c_{e}\right)+\frac{\beta}{\left|R_{c}\right|} \sum_{e \in R_{c}} c_{e}
$$

where $\alpha$ and $\beta$ are user-defined parameters, and $c_{e}$ denotes the congestion of edge $e$, which is defined by

$$
c_{e}=d_{e} / p_{e}
$$

where $d_{e}$ and $p_{e}$ are the density and capacity associated with $e$, respectively. By dynamic density, pattern routing uses an L-shaped (one-bend) or Z-shaped (two-bend) route to make the connection, which gives the shortest path length between two points. Therefore, the wire length is minimum; thus, we do not include it in the cost function at this stage. This cost function can guide our global router to select a path with smaller maximum and average congestion. Note that density $d_{e}$ in (2) comes from both the predicted congestion obtained at the prerouting stage and real routing. Its value is dynamically updated as the routing proceeds.

Fig. 5 illustrates the effects of the prerouting-guided global routing. Fig. 5(a) gives a global-routing instance with four nets $n_{1}, n_{2}, n_{3}$, and $n_{4}$. A traditional bottom-up net-ordered router would route the nets in the order $n_{1}, n_{2}, n_{3}$, and $n_{4}$ based on their net lengths in each level, from short to long, as shown in Fig. 5(b). With the prerouting-guided congestion-driven global routing and iterative refinement, in contrast, we can proceed with the routing, as shown in Fig. 5(c), and obtain a better solution.

\section{Redundant-Via Aware Detailed Routing Stage}

Similar to the global-routing stage, the second bottom-up detail-routing pass is a coarsening process starting from the finest level to the coarsest level. In addition to the routability consideration, we shall also maximize the possibility for postlayout redundant-via insertion in this stage. To do so, Xu et al. [26] considered via minimization and redundant-via planning during detailed routing. They assigned redundant-via costs to the edges of the detailed routing graph to estimate the number of dead vias induced by the route and applied Lagrangian relaxation to solve the problem. However, their cost assignment is not suitable for gridless routing, because there exists no uniform grid for gridless routing, and the high time complexity of Lagrangian relaxation limits their applications to only hundreds of nets.

To simultaneously consider redundant-via planning and via minimization, we define the following cost function for a net $n$ to guide the maze routing:

$$
\Phi_{n}=\gamma \nu(n)+\delta \rho(n)
$$

where $\gamma$ and $\delta$ are user-defined parameters, $\nu(n)$ is the number of vias in $n$, and $\rho(n)$ is a redundant-via related penalty function for $n$. 


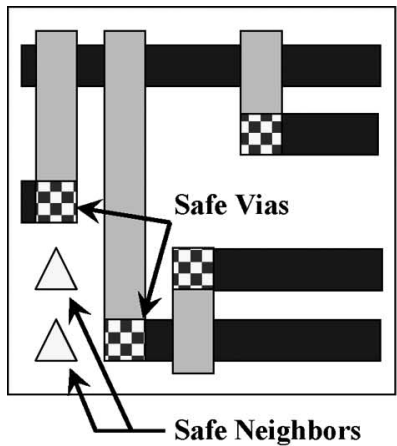

(a)

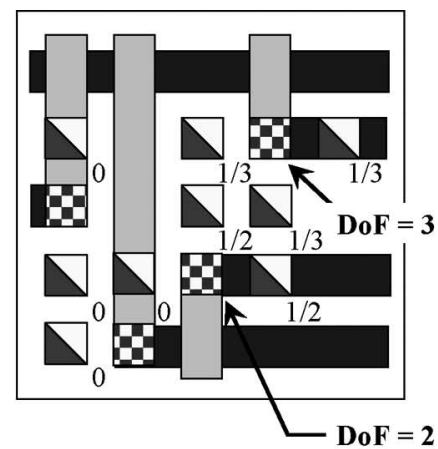

(b)

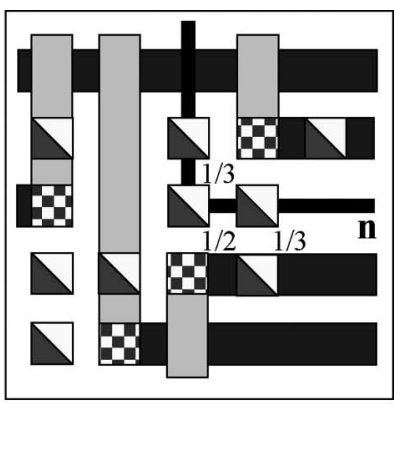

(c)

$\square$ Metal 1

Metal 2 Via

Redundant-Via Candidate

Fig. 6. Redundant-via related penalty assignment. (a) Safe neighbors and safe vias. (b) Cost assignments. (c) Penalty function of net $n$ equals $7 / 6$.

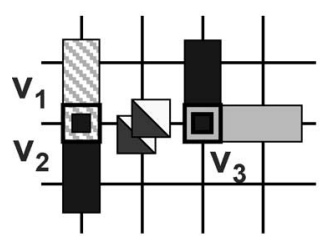

routing layout

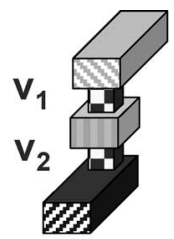

cross-section view

(a)
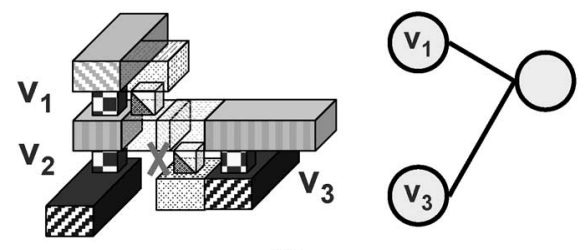

(b)

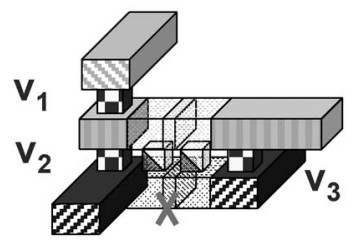

(c)
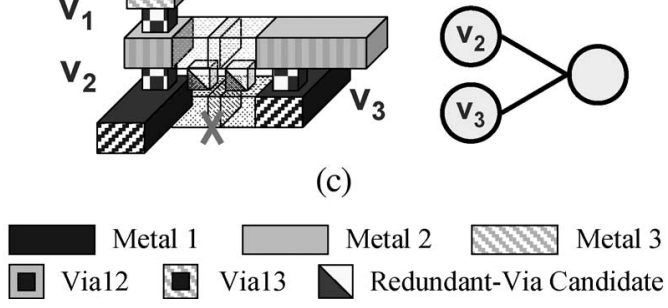

Fig. 7. Counterexample for maximum bipartite matching formulation. (a) Routing layout consists of three single vias $v_{1}, v_{2}$, and $v_{3}$. (b) Vertical design-rule conflict exists between the redundant-via candidates of $v_{1}$ and $v_{3}$. (c) Horizontal design-rule conflict exists between the redundant-via candidates of $v_{2}$ and $v_{3}$.

In this stage, we perform the modified Lee's detailed maze routing algorithm by selecting the paths with the minimum cost of (3) among all the shortest paths. We explain the determination of $\rho(n)$. We call a redundant-via candidate a safe neighbor if it is always available (e.g., it has a special position such that it never vanishes due to other nets passing through it) and is not shared with any other via. A via with at least one safe neighbor is called a safe via [see Fig. 6(a)]. Since a safe via can always be protected by its safe neighbor, we set the cost to zero in all its redundant-via candidates. The degree of freedom of a via $v$, which is denoted by $\operatorname{DoF}_{v}$, is the total number of redundant-via candidates of $v$, as defined in [26]. If via $v$ is not a safe via, we assign the cost $1 / \operatorname{DoF}_{v}$ to its

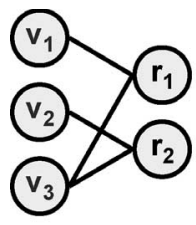

(a)

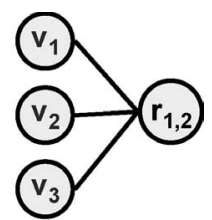

(b)

Fig. 8. Some possible bipartite graph formulations for Fig. 7(a). (a) Infeasible bipartite graph formulation. (b) Feasible bipartite graph formulation but cannot achieve the optimal solution.

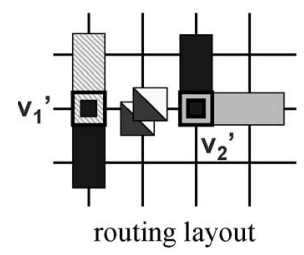

$$
\text { (a) }
$$

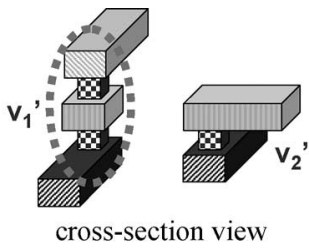

(a)

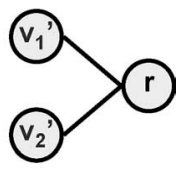

(b)

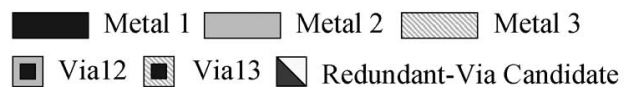

Fig. 9. (a) Stacked-via structure. (b) Our bipartite graph formulation.

redundant-via candidates. The rational is that, if via $v$ becomes a dead via due to net $n$ (i.e., $n$ passes all the redundant-via candidates of $v$ ), the cost for routing $n$ would be increased by $\operatorname{DoF}_{v} \times 1 / \operatorname{DoF}_{v}=1$, which is exactly equal to the number of the induced dead vias. Note that a redundant-via candidate $r_{c}$ may be shared by more than one via. In this case, we set the cost of $r_{c}$ as $\left\{\max \left\{1 / \operatorname{DoF}_{v_{i}}\right\} \mid v_{i}\right.$ is the via that shares $\left.r_{c}\right\}$. Fig. 6(b) shows the redundant-via candidates and their cost assignments. Finally, the penalty function $\rho(n)$ of net $n$ is the summation of the costs of the redundant-via candidates that are passed through by $n$. The $\rho(n)$ of route $n$ in Fig. 6(c) is calculated as $1 / 3+1 / 2+1 / 3=7 / 6$.

\section{PDVI}

Yao et al. [27] claimed that the PDVI problem can be straightforwardly solved by a maximum bipartite matching formulation, which was recently shown to be incorrect in [18]. Fig. 7(a) shows a counterexample consisting of three single vias $v_{1}, v_{2}$, and $v_{3}$. Note that $v_{1}$ and $v_{2}$ belong to the same net, which is different from the net of $v_{3}$. We can see from Fig. 7(b) that 


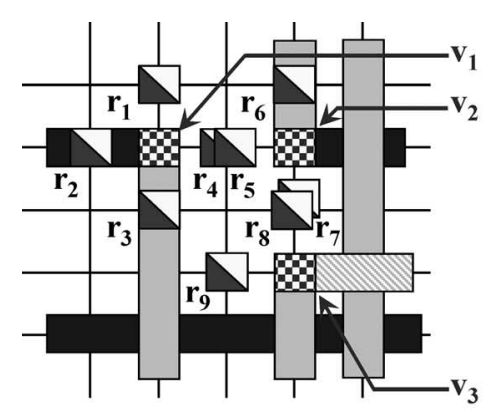

(a)

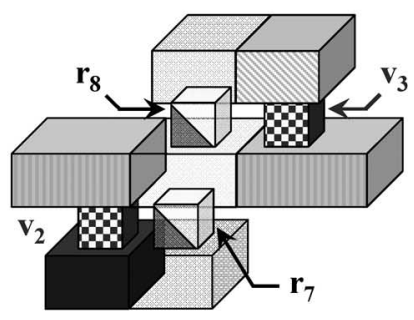

(b)

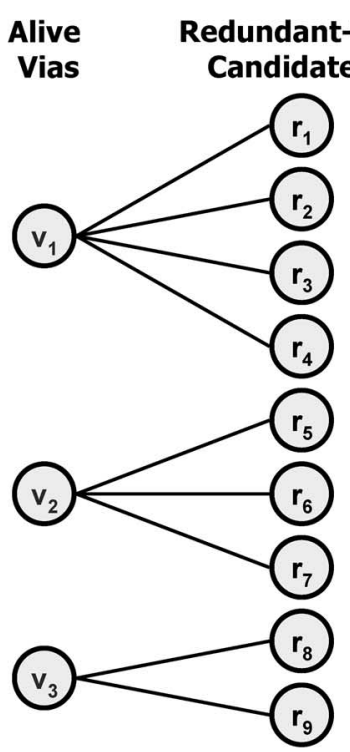

(c)

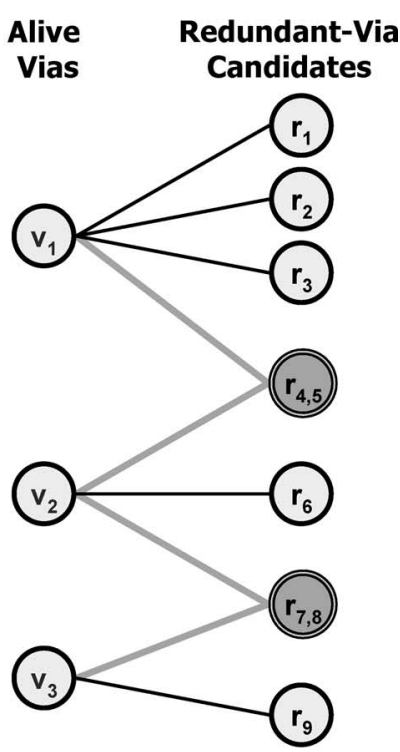

(d)

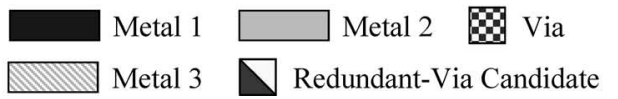

Fig. 10. Bipartite graph construction. (a) A grid-based routing layout. (b) Cross-sectional view for the vertical design-rule conflict between $r_{7}$ and $r_{8}$. (c) Initial bipartite graph without considering conflicts. (d) Final bipartite graph after merging $\left(r_{4}, r_{5}\right)$ and $\left(r_{7}, r_{8}\right)$.

there is a vertical design-rule conflict between the redundantvia candidates of $v_{1}$ and $v_{3}$. Therefore, vias $v_{1}$ and $v_{3}$ cannot simultaneously be paired with a redundant via, or these two nets will be short together. Thus, in the bipartite graph, $v_{1}$ and $v_{3}$ have to connect to the same node to model the constraint. Similarly, vias $v_{2}$ and $v_{3}$ cannot simultaneously be paired with a redundant via because a horizontal design-rule conflict exists between their redundant-via candidates, so they also connect to one node, as shown in Fig. 7(c).

To get the whole bipartite graph, we can simply combine the bipartite graphs together, as shown in Fig. 8(a) and (b). However, a maximum bipartite matching solution of Fig. 8(a) may lead to an infeasible solution that $v_{1}$ and $v_{3}$ are simultaneously paired with a redundant via, which is a contradiction to Fig. 7(b). On the other hand, although all maximum bipartite matching solutions of the formulation in Fig. 8(b) are feasible, these solutions cannot obtain the optimal solution in which $v_{1}$ and $v_{2}$ can be simultaneously paired. As a result, Lee and Wang [18] instead formulated the double-via insertion problem as a MIS problem. Since the MIS problem is NP-complete [15], they resorted to heuristics to handle the problem.

For the PDVI (Post-layout Double-Via Insertion) problem, we develop a polynomial-time double-via insertion algorithm based on a bipartite graph matching formulation. In particular, this graph matching based algorithm is optimal for grid-based routing with up to three routing layers (i.e., two via layers) and the stacked-via structure [i.e., two or more vias vertically stacked are treated as one stacked via; see $v_{1}^{\prime}$ in Fig. 9(a)]. With the algorithm for the restricted problem, we then extend it to handle the general case of any number of routing layers and via structure. With our method, the counterexample shown in [18] can be exactly formulated, as shown in Fig. 9(b). The maximum bipartite matching solution will be either stacked

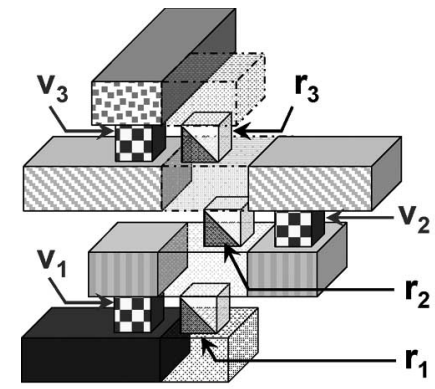

(a)

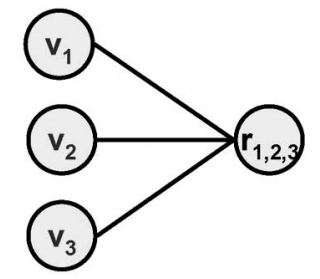

(b)

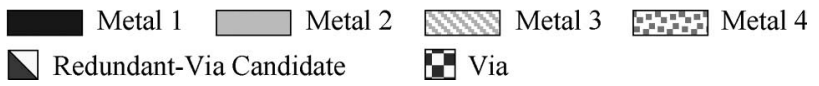

Fig. 11. (a) Four-layer routing layout. (b) The bipartite graph cannot achieve the optimal solution.

via $v_{1}^{\prime}$ is paired with a stacked redundant via or single via $v_{2}^{\prime}$ is paired with a redundant via.

\section{A. Optimal Algorithm for Up to Three Routing Layers}

Our bipartite graph construction is given as follows: Given a routing layout with up to three layers, we first construct an undirected bipartite graph $G(V, E)$, with two disjoint vertex partitions $V_{A}$ and $V_{C}$ such that $V=V_{A} \cup V_{C}$. Here, $V_{A}$ is the set of alive vias, and $V_{C}$ is the set of redundant-via candidates. For $v_{a} \in V_{A}$ and $r_{c} \in V_{C}$, an edge $\left(v_{a}, r_{c}\right) \in E$ exists if $r_{c}$ is a redundant-via candidate of $v_{a}$. Next, we merge two nodes $r_{i}, r_{j} \in V_{C}$ into one node $r_{i, j}$ if there is a vertical or horizontal design-rule conflict between the redundant-via candidates $r_{i}$ and $r_{j}$ (i.e., $r_{i}$ and $r_{j}$ cannot be simultaneously inserted). For example, Fig. 10(a) gives a grid-based routing layout, where a horizontal conflict exists between $r_{4}$ and $r_{5}$ and a vertical 


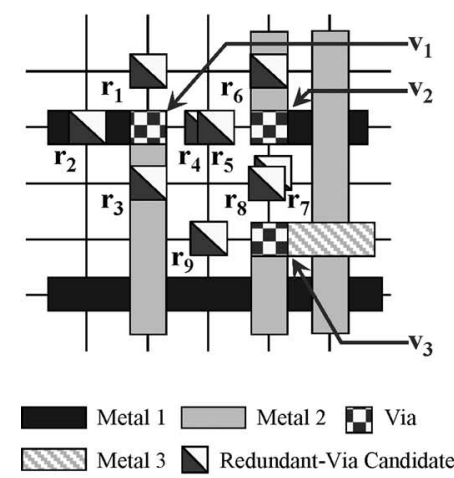

(a)

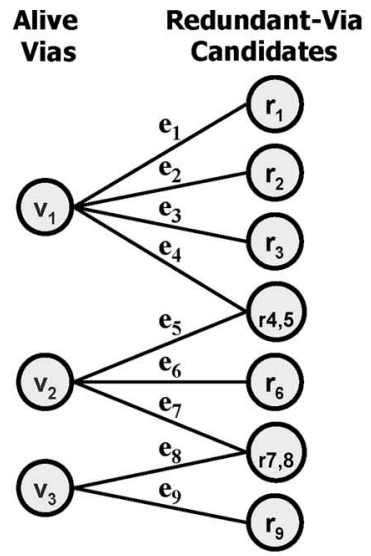

(b)

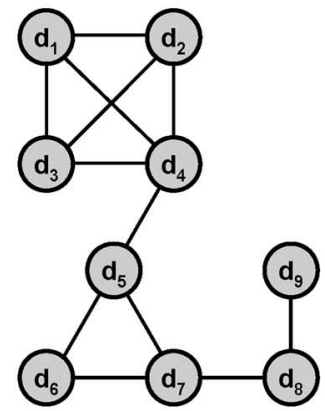

(c)

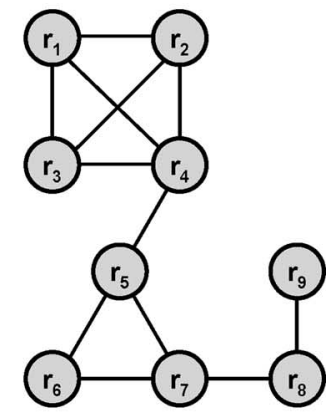

(d)

Fig. 12. Illustration of the proof for Theorem 1. (a) Postrouting layout. (b) Bipartite graph. (c) Line graph constructed from the bipartite graph. (d) Conflict graph constructed from the layout.

conflict exists between $r_{7}$ and $r_{8}$. Fig. 10(b) shows the crosssectional view of the vertical design-rule conflict between $r_{7}$ and $r_{8}$. Fig. 10(c) gives the initial bipartite graph construction, and Fig. 10(d) presents the final bipartite graph after merging $r_{4}$ and $r_{5}$ into $r_{4,5}$ and merging $r_{7}$ and $r_{8}$ into $r_{7,8}$. For this modeling, a matching $\left(v_{a}, r_{c}\right) \in E$ in the bipartite graph will represent that an alive via $v_{a} \in V_{A}$ is paired with a redundantvia candidate $r_{c} \in V_{C}$ in the resulting routing layout. Notice that this approach readily extends to more general cases since the bipartite graph construction can be applied to both gridless and grid-based layouts.

The reason for the three-routing layers constraint is that, if we have a four-layer routing layout, the constructed bipartite graph may not lead to the optimal solution. For example, Fig. 11(a) shows a four-layer routing layout, and its bipartite graph is shown in Fig. 11(b). We can see that the optimal solution for double-via insertion is to insert a redundant via for each of vias $v_{1}$ and $v_{3}$, which cannot be obtained from the bipartite graph of Fig. 11(b).

We have the following lemmas and theorem showing the optimality of the bipartite graph formulation for grid-based routing with up to three routing layers and the stacked-via structure.

1) Definition 1: Conflict Graph: A conflict graph $C\left(V_{G}, E_{G}\right)$ is an undirected graph constructed from a detailed routing solution. A vertex $r \in V_{G}$ corresponds to a redundant-via candidate in the layout. An edge $\left(r_{i}, r_{j}\right) \in E_{G}$ exists iff both $r_{i}$ and $r_{j}$ are redundant-via candidates of the same via, or $r_{i}$ and $r_{j}$ will cause design-rule conflicts if they both exist.

2) Definition 2: Line Graph: The line graph of a graph $G$, which is denoted by $L(G)$, is a simple graph whose vertices are the edges of $G$, and $\left(u, u^{\prime}\right)$ is an edge of $L(G)$ iff $u$ and $u^{\prime}$ share a vertex of $G$.

Lemma 1: The PDVI problem can be reduced into a MIS problem.

Proof: In the conflict graph $C\left(V_{G}, E_{G}\right)$ of a routing layout, all vertices represent redundant-via candidates. The MIS of $C$, which is denoted by $I$, is a maximum vertex set such that, for any vertex pair $r_{i}, r_{j} \in I,\left(r_{i}, r_{j}\right) \notin E_{C}$. It means that all redundant-via candidates corresponding to vertices in $I$ can be inserted by a redundant via at the same time without incurring any design-rule conflict, and for each via, only one of its redundant-via candidates is chosen for insertion. In addition, because the size of the independent set $|I|$ is the maximum, the number of inserted double vias is also the maximum. Therefore, the PDVI problem can be transformed to a MIS problem.

Lemma 2: Given a grid-based routing layout, the line graph of a bipartite graph formulating the PDVI problem, with up to three routing layers and the stacked-via structure, is isomorphic to the conflict graph of the routing layout. .

Proof: Given a bipartite graph $G_{B}\left(V_{B}, E_{B}\right)$ with the vertex set $V_{B}=V_{A} \cup V_{C}$, where $V_{A}$ is the set of alive vias and $V_{C}$ is the set of redundant-via candidates, we can construct its line graph $L\left(G_{B}\right)=\left(V_{L}, E_{L}\right)$. A vertex $d_{i} \in V_{L}$ corresponds to an edge $e_{i} \in E_{B}$. It is clear that each vertex $d_{i} \in V_{L}$ in $L\left(G_{B}\right)$ corresponds to a vertex $r_{i}$ in the conflict graph $C\left(V_{G}, E_{G}\right)$. By Definition 2, an edge $\left(d_{i}, d_{j}\right) \in E_{L}$ exists iff, in $G_{B}$ :

(1) $e_{i}$ and $e_{j}$ share the same vertex $v_{k} \in V_{A}$, or

(2) $e_{i}$ and $e_{j}$ share the same vertex $r_{k} \in V_{C}$.

It is clear that $\left(d_{i}, d_{i+1}, \ldots, d_{p}\right) \in E_{L}$ forms a clique if edges $\left(e_{i}, e_{i+1}, \ldots e_{p}\right)$ share the same vertex $v_{k} \in V_{A}$ or $r_{k} \in V_{C}$. For Case (1), it means that $r_{i}$ and $r_{j}$ are redundant-via candidates of the same single via, where $r_{i}$ and $r_{j}$ are redundantvia candidates incident by $e_{i}$ and $e_{j}$, respectively. Thus, there also exists such an edge $\left(r_{i}, r_{j}\right) \in E_{G}$. Besides, redundantvia candidates of the same single via also form a clique in the conflict graph. For Case (2), it means that $r_{i}$ and $r_{j}$ have horizontal or vertical design-rule conflicts, and thus there also exists such an edge $\left(r_{i}, r_{j}\right) \in E_{G}$. For the redundant-via candidates in grid-based routing with up to three routing layers and the stacked-via structure, they also form a clique with conflict edges only. So the line graph induced from our bipartite graph is isomorphic to the conflict graph of the routing layout under the same assumption. See Fig. 12 for an example.

Lemma 3: A maximum matching in the formulated bipartite graph corresponds to a MIS in the conflict graph. 


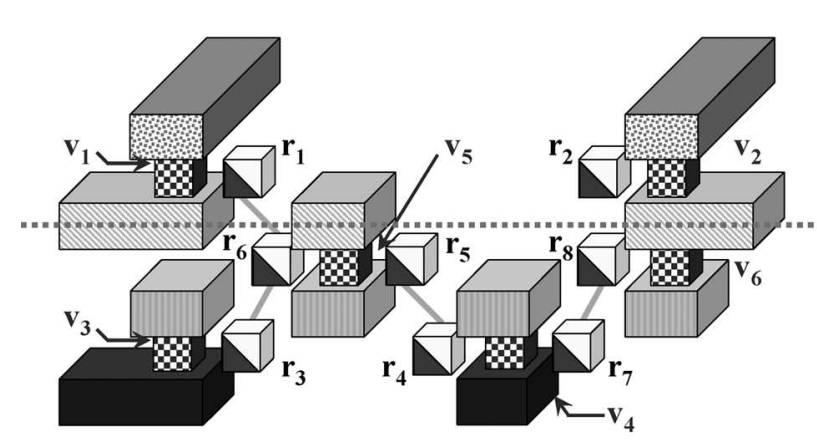

(a)

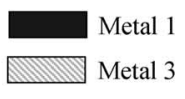

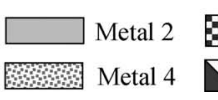

Redundant-Via Candidate
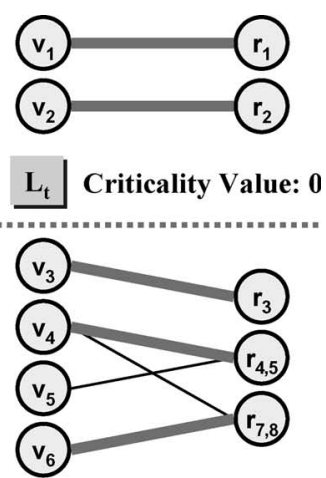

$L_{b}$ Criticality Value: 2

(b)

Fig. 13. Illustration of the TDVI algorithm. (a) Cross-sectional view of the whole layout. (b) Bipartite graphs of the subproblems.

Proof: Independent set in its line graph [23]: Thus, a maximum matching in $G_{B}$ is an independent set in $L\left(G_{B}\right)$, in which the number of vertices is the maximum. Then, according to Lemma $2, L\left(G_{B}\right)$ is isomorphic to conflict graph $C$. As a result, we can prove that a maximum matching solution in bipartite graph $G_{B}$ is a MIS in conflict graph $C$.

Theorem 1: The maximum bipartite matching algorithm optimally solves the PDVI problem (i.e. the number of inserted double vias is maximum) for grid-based routing, with up to three routing layers (i.e., two via layers) and the stacked-via structure, in $O(\sqrt{|V|}|E|)$ time, where $V$ is the set of vertices and $E$ is the set of edges in the bipartite graph $G=(V, E)$.

Proof: By Lemmas 1-3 the PDVI problem with up to three routing layers can be exactly reduced into a maximum bipartite matching problem by constructing a bipartite graph from the given grid-based routing layout under the stackedvia structure. Let $M$ denote a maximum matching in $G_{B}$. Then, a matching $\left(v_{i}, r_{j}\right) \in M$, where $v_{i} \in V_{A}$ and $r_{j} \in V_{C}$, represents that redundant-via candidate $r_{j}$ is inserted for single via $v_{i}$. Through the bipartite maximum matching formulation, we can get a maximum matching solution, so that the number of double vias inserted is maximum, which is optimal. By applying Hopcroft and Karp's algorithm [23], a maximum matching could be found in $O(\sqrt{|V|}|E|)$ time. As a result, the theorem is established.

\section{B. On-Track/Stacked Redundant-Via Enhancement}

In the redundant-via insertion process, redundant vias can be placed on-track or off-track. A redundant via $r$ of a via $v$ is on-track if $r$ is placed on the wire segment of $v$; it is offtrack otherwise. In Fig. 10, redundant-via candidates $r_{2}$ and $r_{3}$ are on-track, whereas $r_{1}$ and $r_{4}$ are off-track. Since on-track redundant vias consume fewer routing resources than off-track ones, we prefer selecting on-track redundant-via candidates for insertion. Furthermore, we can also give higher priority to the redundant-via candidates of stacked vias to improve the reliability since stacked vias are more defect-prone and thus more desired for protection than single vias.

We formulate the PDVI problem considering on-/off-track redundant vias and stacked vias as a minimum weighted bipar- tite matching problem. The construction of vertices and edges is the same as Section IV-A, whereas the edge weight $w(v, r)$ of an edge $(v, r) \in E$ is given by

$$
\begin{aligned}
w(v, r) & =\left\{\begin{array}{lc}
\frac{t_{r}}{n_{s}}, & \text { if } v \text { is a stacked via } \\
t_{r}, & \text { otherwise }
\end{array}\right. \\
t_{r} & = \begin{cases}1, & \text { if } r \text { is on-track } \\
2, & \text { if } r \text { is off-track }\end{cases}
\end{aligned}
$$

With this weighting policy, the minimum weighted bipartite matching will give us a solution that prefers more on-track redundant vias and provides more protection for stacked vias.

\section{TDVI Algorithm}

For the general case with any number of routing layers, we propose a two-stage double-via insertion (TDVI) algorithm by extending the algorithm for up to three routing layers and the stacked-via structure.

First, we partition the original layout into sublayouts composed of up to three routing layers each, with the objective to minimize the number of vertical design-rule conflicts between sublayouts. Every redundant-via candidate $r_{c}$ is associated with a criticality $c_{r}$. If $r_{c}$ has a vertical design-rule conflict with some redundant-via candidates lying in the different sublayouts, then $c_{r}$ is equal to the number of induced dead vias if $r_{c}$ is inserted; $c_{r}$ is equal to zero otherwise. The criticality value of a sublayout is the summation of the criticalities of the redundant-via candidates inside it. We then find solutions for the sublayouts one by one in the nondecreasing order of the criticality value. The sublayout with a lower criticality value has higher priority for processing since it contains more critical vias adjacent to the cut lines. During the subproblem solving stage, if we prefer selecting on-track/stacked redundant vias, the subproblem is formulated as a minimum weighted bipartite matching problem, as in Section IV-B; otherwise, it is solved by maximum bipartite matching, as in Section IV-A, to maximize the insertion rate. After solving one sublayout $L_{i}$, all sublayouts adjacent to $L_{i}$ need to be updated by removing the infeasible redundant-via candidates. Continuing with the process, we can obtain the final solution for the original layout. 


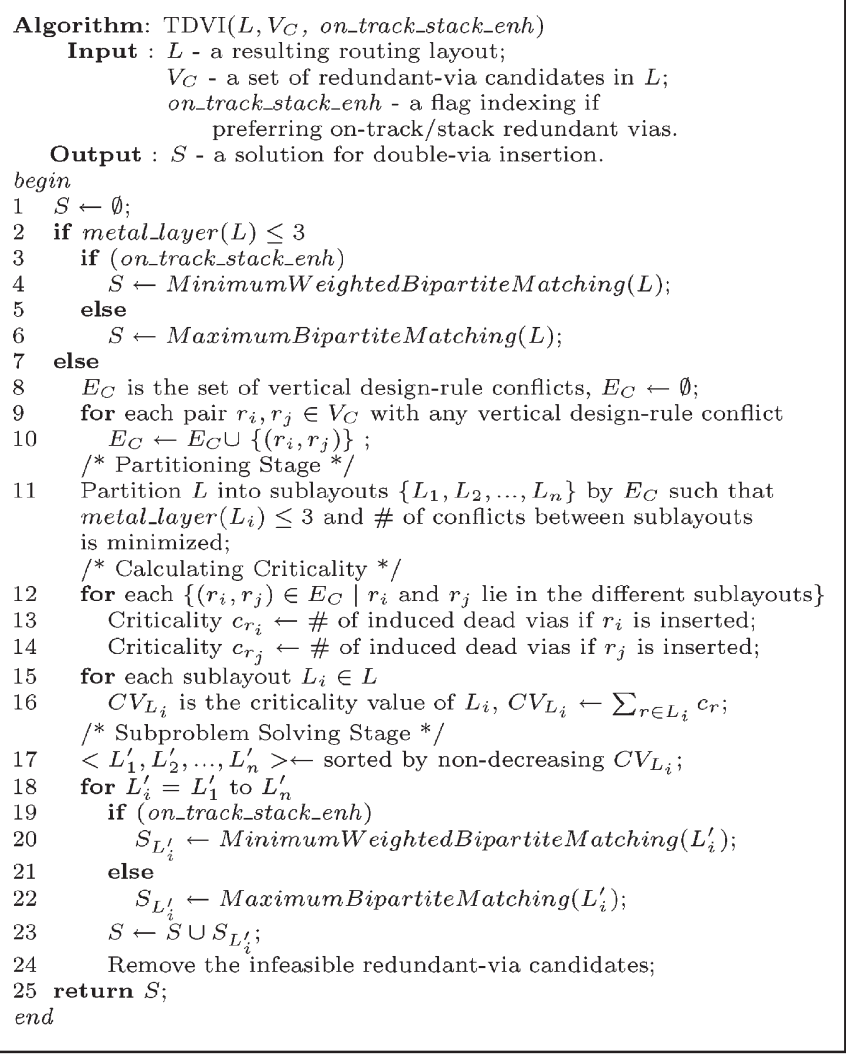

Fig. 14. TDVI algorithm.

For the example shown in Fig. 13, the four-layer routing layout is partitioned into two sublayouts $L_{t}$ and $L_{b}$ with a vertical design-rule conflict between them. The redundant-via candidate $r_{1}$ in $L_{t}$ has a vertical design-rule conflict, with $r_{6}$ lying in the different sublayout $L_{b}$, and there is no induced dead vias if we insert $r_{1}$. Therefore, $c_{1}$ is equal to 0 . Similarly, $r_{6}$ in $L_{b}$ has a vertical design-rule conflict, with $r_{1}$ lying in the different sublayout $L_{t}$, and two vias $v_{1}$ and $v_{3}$ will become dead vias if we insert $r_{6}$. Thus, $c_{6}$ is equal to 2 . As a result, the criticality values of sublayouts $L_{t}$ and $L_{b}$ are equal to zero and two, respectively. Then, the two-layer sublayout $L_{t}$ is processed before the three-layer sublayout $L_{b}$ since $L_{t}$ has lower criticality value than $L_{b}$. After getting the solution $\left\{\left(v_{1}, r_{1}\right),\left(v_{2}, r_{2}\right)\right\}$ for $L_{t}, L_{b}$ is updated by removing the infeasible redundant-via candidate $r_{6}$. After getting the solution $\left\{\left(v_{3}, r_{3}\right),\left(v_{4}, r_{4}\right),\left(v_{6}, r_{8}\right)\right\}$ for $L_{b}$, the final solution for the whole layout is $\left\{\left(v_{1}, r_{1}\right),\left(v_{2}, r_{2}\right),\left(v_{3}, r_{3}\right),\left(v_{4}, r_{4}\right),\left(v_{6}, r_{8}\right)\right\}$. The TDVI algorithm is summarized in Fig. 14.

Note that, for the maximization of the redundant-via insertion rate, the TDVI algorithm is optimal for grid-based routing if no conflicts exist between the partitioned sublayouts, since each sublayout can be solved optimally with the maximum bipartite matching.

\section{EXPERIMENTAL RESULT}

The TBR algorithm has been implemented using the C++ programming language on a $1.2-\mathrm{GHz}$ SUN Blade-2000 workstation with 8-GB memory.
We perform the routing algorithm on the MCNC benchmarks (provided by Cong et al. [10]) and the PDVI on both the MCNC and the industrial Faraday benchmarks (introduced in [1]). We consider the design rules of wire width, wire spacing, wire pitch, via width, and via spacing provided in these designs.

Tables I and II list the set of benchmark circuits. In the table, "Circuit" gives the names of the circuits, "Size" gives the layout dimensions in micrometer square, "\#Layer" denotes the number of routing layers used, "\#Net" gives the total number of nets, "\#Connections" gives the number of two-pin connections after net decomposition, and "\#Pin" gives the number of pins. The results can be downloaded from the web site http://cc.ee.ntu.edu.tw/ ywchang/research.html.

\section{A. Routability-Driven Routing Framework}

We compared TBR with three state-of-the-art gridless routers: 1) the $\Lambda$-shaped multilevel router MGR [8];2) the multilevel global routing + flat gridless detailed routing system (MARS) [10]; and 3) the V-shaped multilevel router (VMGR) [9]. MGR and VMGR were provided by Chen and Chang [8] and Chen et al. [9]. The experimental results on the single via count, routing completion rate, and runtime are listed in Table III. Here, the four routers were all performed in the routability-driven mode. MGR, VMGR, and TBR were run on the same machine, whereas the results of MARS were directly taken from [10]. (Note that [10] does not report the via count for MARS, and MARS was run on a 440-MHz SUN Ultra-10 workstation. We tried our best to make a fair comparison by normalizing its runtime by the factor $440 / 1200$ based on the clock rates and reported the normalized results in Table III.) As shown in the table, all the four routers obtain $100 \%$ routing completion, whereas TBR achieves about $7.2 \times, 2.6 \times$, and $1.4 \times$ runtime speedups compared with MGR, MARS, and VMGR, respectively. Furthermore, TBR reduces the single-via count by 20\% and 23\% over MGR and VMGR, respectively.

\section{B. Double-Via Aware Detailed Routing}

We also performed experiments on double-via aware gridless detailed routing. Table IV shows the routing results of TBR with and without double-via planning during detailed routing. In the table, "\#Total Via" gives the total number of vias in the routing result, "\#Dead Via" gives the number of dead vias, "\#Critical Via" denotes the number of critical vias, and "\#WL" reports the total wire length. The experimental results show that the redundant-via aware detailed routing results in fewer dead vias and critical vias by factors of 1.44 and 1.14, respectively, using similar running times. The slight increase in the via count (2\%) is as expected, because the detailed router has to make detours not to incur more critical and dead vias. The small overhead on the via count also reflects the effectiveness of the via control by (3).

\section{PDVI}

We implemented the $\mathrm{H} 2 \mathrm{~K}$ and $\mathrm{H} 3 \mathrm{~K}$ algorithms proposed in [18] under the stacked-via structure, and named them H2K_S and H3K_S respectively. We compared our TDVI algorithm 
TABLE I

MCNC BENCHMARK CIRCUITS

\begin{tabular}{|c|c|c|c|c|c|}
\hline Circuit & Size $\left(\mu \mathrm{m}^{2}\right)$ & \#Layers & \#Nets & \#Connections & \#Pins \\
\hline Mcc1 & $45000 \times 39000$ & 4 & 802 & 1693 & 3101 \\
Mcc2 & $152400 \times 152400$ & 4 & 7118 & 7541 & 25024 \\
Struct & $4903 \times 4904$ & 3 & 1920 & 3551 & 5471 \\
Primary1 & $7522 \times 4988$ & 3 & 904 & 2037 & 2941 \\
Primary2 & $10438 \times 6488$ & 3 & 3029 & 8197 & 11226 \\
S5378 & $435 \times 239$ & 3 & 1694 & 3124 & 4818 \\
S9234 & $404 \times 225$ & 3 & 1486 & 2774 & 4260 \\
S13207 & $660 \times 365$ & 3 & 3781 & 6995 & 10776 \\
S15850 & $705 \times 389$ & 3 & 4472 & 8321 & 12793 \\
S38417 & $1144 \times 619$ & 3 & 11309 & 21035 & 32344 \\
S38584 & $1295 \times 672$ & 3 & 14754 & 28177 & 42931 \\
\hline
\end{tabular}

TABLE II

FARADAY BENCHMARK CIRCUITS

\begin{tabular}{|c|c|c|c|c|c|}
\hline Circuit & Size $\left(\mu \mathrm{m}^{2}\right)$ & \#Layers & \#Nets & \#Connections & \#Pins \\
\hline DMA & $408.4 \times 408.4$ & 6 & 13256 & 36162 & 73982 \\
DSP1 & $706 \times 706$ & 6 & 28447 & 63495 & 144872 \\
DSP2 & $642.8 \times 642.8$ & 6 & 28431 & 36686 & 144703 \\
RISC1 & $1003.6 \times 1003.6$ & 6 & 34034 & 95106 & 196677 \\
RISC2 & $959.6 \times 959.6$ & 6 & 34034 & 95099 & 196670 \\
\hline
\end{tabular}

TABLE III

COMPARISON FOR GRIDLESS ROUTERS

\begin{tabular}{|c|c|c|c|c|c|c|c|c|c|c|c|}
\hline \multirow[b]{2}{*}{ Circuit } & \multicolumn{3}{|c|}{ MGR [8] } & \multicolumn{2}{|c|}{ MARS [10] } & \multicolumn{3}{|c|}{ VMGR [9] } & \multicolumn{3}{|c|}{ TBR (Ours) } \\
\hline & $\begin{array}{c}\text { \#Single } \\
\text { Via }\end{array}$ & $\begin{array}{l}\text { Cmp. } \\
\text { Rate }\end{array}$ & $\begin{array}{l}\text { Time } \\
(\mathrm{sec})\end{array}$ & $\begin{array}{l}\text { Cmp. } \\
\text { Rate }\end{array}$ & $\begin{array}{l}\text { Time } \\
(\mathrm{sec})\end{array}$ & $\begin{array}{c}\text { \#Single } \\
\text { Via }\end{array}$ & $\begin{array}{l}\text { Cmp. } \\
\text { Rate }\end{array}$ & $\begin{array}{l}\text { Time } \\
\text { (sec) }\end{array}$ & $\begin{array}{c}\text { \#Single } \\
\text { Via }\end{array}$ & $\begin{array}{l}\text { Cmp. } \\
\text { Rate }\end{array}$ & $\begin{array}{l}\text { Time } \\
\text { (sec) }\end{array}$ \\
\hline Mcc1 & 5791 & $100 \%$ & 171.2 & $100 \%$ & 38.8 & 5765 & $100 \%$ & 51.5 & 5788 & $100 \%$ & 25.7 \\
\hline $\mathrm{Mcc} 2$ & 33093 & $100 \%$ & 3339.9 & $100 \%$ & 702.9 & 34387 & $100 \%$ & 1149.1 & 33153 & $100 \%$ & 783.0 \\
\hline Struct & 10055 & $100 \%$ & 5.9 & $100 \%$ & 11.6 & 9969 & $100 \%$ & 3.5 & 7248 & $100 \%$ & 2.8 \\
\hline Primary 1 & 6374 & $100 \%$ & 4.6 & $100 \%$ & 12.3 & 6169 & $100 \%$ & 4.2 & 5347 & $100 \%$ & 2.7 \\
\hline Primary2 & 25955 & $100 \%$ & 42.0 & $100 \%$ & 59.7 & 24977 & $100 \%$ & 23.7 & 22365 & $100 \%$ & 17.7 \\
\hline S5378 & 8296 & $100 \%$ & 41.0 & $100 \%$ & 11.0 & 8520 & $100 \%$ & 4.6 & 6784 & $100 \%$ & 4.0 \\
\hline S9234 & 6833 & $100 \%$ & 22.6 & $100 \%$ & 8.4 & 6690 & $100 \%$ & 3.4 & 5350 & $100 \%$ & 2.9 \\
\hline S13207 & 17127 & $100 \%$ & 122.6 & $100 \%$ & 31.2 & 18263 & $100 \%$ & 15.7 & 13767 & $100 \%$ & 11.6 \\
\hline $\mathrm{S} 15850$ & 19875 & $100 \%$ & 326.0 & $100 \%$ & 39.3 & 23405 & $100 \%$ & 20.0 & 16633 & $100 \%$ & 16.4 \\
\hline S38417 & 50304 & $100 \%$ & 362.8 & $100 \%$ & 92.0 & 52798 & $100 \%$ & 55.9 & 40655 & $100 \%$ & 43.7 \\
\hline S38584 & 67026 & $100 \%$ & 688.6 & $100 \%$ & 170.9 & 74054 & $100 \%$ & 191.6 & 54483 & $100 \%$ & 148.5 \\
\hline Comp. & 1.20 & $100 \%$ & 7.15 & $100 \%$ & 2.59 & 1.23 & $100 \%$ & 1.37 & 1.00 & $100 \%$ & 1.00 \\
\hline
\end{tabular}

TABLE IV

REDUNDANT-Via AWARE GRIDLESS Routing

\begin{tabular}{|c|c|c|c|c|c|c|c|c|c|c|c|c|}
\hline \multirow[b]{2}{*}{ Circuit } & \multicolumn{6}{|c|}{ Without Redundant Via Consideration } & \multicolumn{6}{|c|}{ With Redundant Via Consideration } \\
\hline & $\begin{array}{c}\text { Comp. } \\
\text { Rate }\end{array}$ & $\begin{array}{c}\text { \#Total } \\
\text { Via }\end{array}$ & $\begin{array}{c}\text { \#Dead } \\
\text { Via }\end{array}$ & $\begin{array}{c}\text { \#Critical } \\
\text { Via }\end{array}$ & $\begin{array}{c}\text { WL } \\
\text { (um) }\end{array}$ & $\begin{array}{l}\text { Time } \\
(\mathrm{sec})\end{array}$ & $\begin{array}{c}\text { Comp. } \\
\text { Rate }\end{array}$ & $\begin{array}{c}\text { \#Total } \\
\text { Via }\end{array}$ & $\begin{array}{c}\text { \#Dead } \\
\text { Via }\end{array}$ & $\begin{array}{c}\text { \#Critical } \\
\text { Via }\end{array}$ & $\begin{array}{c}\text { WL } \\
\text { (um) }\end{array}$ & $\begin{array}{l}\text { Time } \\
\text { (sec) }\end{array}$ \\
\hline Mcc1 & $100 \%$ & 5371 & 616 & 1546 & $2.817 \mathrm{E}+07$ & 25.7 & $100 \%$ & 5501 & 388 & 1314 & $2.818 \mathrm{E}+07$ & 29.8 \\
\hline Mcc2 & $100 \%$ & 31229 & 4178 & 8739 & $4.055 \mathrm{E}+08$ & 783.0 & $100 \%$ & 32148 & 2617 & 7517 & $4.055 \mathrm{E}+08$ & 997.3 \\
\hline Struct & $100 \%$ & 6746 & 58 & 664 & $8.726 \mathrm{E}+05$ & 2.8 & $100 \%$ & 7081 & 42 & 531 & $8.727 \mathrm{E}+05$ & 3.1 \\
\hline Primary 1 & $100 \%$ & 5163 & 100 & 950 & $1.028 \mathrm{E}+06$ & 2.7 & $100 \%$ & 5312 & 63 & 800 & $1.028 \mathrm{E}+06$ & 3.0 \\
\hline Primary 2 & $100 \%$ & 21754 & 586 & 4296 & $4.202 \mathrm{E}+06$ & 17.7 & $100 \%$ & 22306 & 435 & 3788 & $4.203 \mathrm{E}+06$ & 20.0 \\
\hline S5378 & $100 \%$ & 6416 & 619 & 1649 & $7.485 \mathrm{E}+04$ & 4.0 & $100 \%$ & 6387 & 445 & 1492 & $7.493 \mathrm{E}+04$ & 5.0 \\
\hline S9234 & $100 \%$ & 5121 & 436 & 1282 & $5.548 \mathrm{E}+04$ & 2.9 & $100 \%$ & 5129 & 340 & 1139 & $5.552 \mathrm{E}+04$ & 3.4 \\
\hline S13207 & $100 \%$ & 13223 & 1039 & 3246 & $1.786 \mathrm{E}+05$ & 11.6 & $100 \%$ & 13397 & 787 & 2913 & $1.789 \mathrm{E}+05$ & 14.2 \\
\hline $\mathrm{S} 15850$ & $100 \%$ & 15914 & 1400 & 3945 & $2.214 \mathrm{E}+05$ & 16.4 & $100 \%$ & 16120 & 1079 & 3682 & $2.217 \mathrm{E}+05$ & 20.6 \\
\hline S38417 & $100 \%$ & 39159 & 3294 & 9686 & $4.871 \mathrm{E}+05$ & 43.7 & $100 \%$ & 39345 & 2484 & 8795 & $4.879 \mathrm{E}+05$ & 53.0 \\
\hline S38584 & $100 \%$ & 52154 & 5605 & 13353 & $6.744 \mathrm{E}+05$ & 148.5 & $100 \%$ & 52894 & 3333 & 12185 & $6.756 \mathrm{E}+05$ & 175.3 \\
\hline Comp. & 1.00 & 0.98 & 1.44 & 1.14 & 1.00 & 0.84 & 1.00 & 1.00 & 1.00 & 1.00 & 1.00 & 1.00 \\
\hline
\end{tabular}

with H2K_S, H3K_S, and a state-of-the-art commercial tool for post-layout double-via insertion.

Both H2K_S and H3K_S divide the original graph into subgraphs and apply an MIS solver to solve each subgraph. H2K_S can achieve a higher insertion rate than H3K_S , while $\mathrm{H} 3 \mathrm{~K} \_\mathrm{S}$ is a modified heuristic of $\mathrm{H} 2 \mathrm{~K} \_\mathrm{S}$ and can increase the number of on-track redundant vias. Since $\mathrm{H} 2 \mathrm{~K}$ and $\mathrm{H} 3 \mathrm{~K}$ use qualex-ms [5] as their MIS solver, which is a Linux-based package, we performed the experiment on a Linux personal computer with an Intel Pentium 4 3.2-GHz central processing unit and 3-GB memory. The settings for the subgraph size (set to 1500 ) and the priority queue are the same as [18]. We ran TBR on the benchmarks to generate routing results, which were then fed into H2K_S, H3K_S, a commercial tool, and TDVI for PDVI. Our routing and double-via insertion results both passed the design rule check verification by the Cadence SOC Encounter.

Table $\mathrm{V}$ shows the double-via insertion comparison between TDVI and H2K_S. In the table, "Via Info." gives the total number of vias "\#Total Via" and alive vias "\#Alive Via" 
TABLE V

COMPARISON FOR DOUbLE-VIA INSERTION With H2K

\begin{tabular}{|c|c|c|c|c|c|c|c|c|c|c|c|c|}
\hline \multirow[b]{2}{*}{ Circuit } & \multicolumn{2}{|c|}{ Via Info. } & \multicolumn{5}{|c|}{ H2K_S } & \multicolumn{5}{|c|}{ TDVI (Ours) } \\
\hline & $\begin{array}{c}\text { \#Total } \\
\text { Via }\end{array}$ & $\begin{array}{c}\text { \#Alive } \\
\text { Via }\end{array}$ & $\begin{array}{l}\text { \#Ins. } \\
\text { Rvia }\end{array}$ & $\begin{array}{l}\text { Ins. } \\
\text { Rate }\end{array}$ & $\begin{array}{l}\text { \#On- } \\
\text { Track } \\
\text { Rvia }\end{array}$ & $\begin{array}{l}\text { On- } \\
\text { Track } \\
\text { Rate }\end{array}$ & $\begin{array}{c}\text { Time } \\
\text { (s) }\end{array}$ & $\begin{array}{l}\text { \#Ins. } \\
\text { Rvia }\end{array}$ & $\begin{array}{l}\text { Ins. } \\
\text { Rate }\end{array}$ & $\begin{array}{l}\text { \#On- } \\
\text { Track } \\
\text { Rvia }\end{array}$ & $\begin{array}{l}\text { On- } \\
\text { Track } \\
\text { Rate }\end{array}$ & $\begin{array}{c}\text { Time } \\
\text { (s) }\end{array}$ \\
\hline Mcc1 & 5501 & 5113 & 4932 & $96.5 \%$ & 2318 & $47.0 \%$ & 45.64 & 5011 & $98.0 \%$ & 2100 & $41.9 \%$ & 0.26 \\
\hline Mcc2 & 32148 & 29531 & 28670 & $97.1 \%$ & 12982 & $45.3 \%$ & 395.90 & 29099 & $98.5 \%$ & 12360 & $42.5 \%$ & 1.74 \\
\hline Struct & 7081 & 7039 & 7019 & $99.7 \%$ & 3001 & $42.8 \%$ & 104.44 & 7037 & $99.9 \%$ & 3444 & $48.9 \%$ & 0.23 \\
\hline Primary1 & 5312 & 5249 & 5207 & $99.2 \%$ & 2405 & $46.2 \%$ & 73.08 & 5244 & $99.9 \%$ & 2625 & $50.1 \%$ & 0.16 \\
\hline Primary2 & 22306 & 21871 & 21713 & $99.3 \%$ & 10242 & $47.2 \%$ & 305.64 & 21842 & $99.9 \%$ & 11294 & $51.7 \%$ & 0.75 \\
\hline S5378 & 6387 & 5942 & 5611 & $94.4 \%$ & 2724 & $48.5 \%$ & 59.46 & 5806 & $97.7 \%$ & 2658 & $45.8 \%$ & 0.20 \\
\hline S9234 & 5129 & 4789 & 4543 & $94.9 \%$ & 2271 & $50.0 \%$ & 50.76 & 4710 & $98.4 \%$ & 2113 & $44.9 \%$ & 0.16 \\
\hline S13207 & 13397 & 12610 & 11983 & $95.0 \%$ & 5970 & $49.8 \%$ & 97.58 & 12356 & $98.0 \%$ & 5543 & $44.9 \%$ & 0.43 \\
\hline S15850 & 16120 & 15041 & 14272 & $94.9 \%$ & 7033 & $49.3 \%$ & 132.34 & 14718 & $97.9 \%$ & 6726 & $45.7 \%$ & 0.52 \\
\hline S38417 & 39345 & 36861 & 35093 & $95.2 \%$ & 17469 & $49.8 \%$ & 354.03 & 36170 & $98.1 \%$ & 16188 & $44.8 \%$ & 1.37 \\
\hline S38584 & 52894 & 49561 & 46823 & $94.5 \%$ & 23044 & $49.2 \%$ & 427.87 & 48478 & $97.8 \%$ & 21954 & $45.3 \%$ & 1.98 \\
\hline \multicolumn{3}{|c|}{ Comp. } & 0.98 & $96.4 \%$ & - & $47.7 \%$ & 299.26 & 1.00 & $98.6 \%$ & - & $46.0 \%$ & 1.00 \\
\hline
\end{tabular}

TABLE VI

COMPARISON FOR DOUble-Via INSERTION With H3K (TDVI RUNS With ON-Track/STACKEd REDUndant-Via ENHANCEMENT)

\begin{tabular}{|c|c|c|c|c|c|c|c|c|c|c|c|c|}
\hline \multirow[b]{2}{*}{ Circuit } & \multicolumn{2}{|c|}{ Via Info. } & \multicolumn{5}{|c|}{ H3K_S } & \multicolumn{5}{|c|}{ TDVI (Ours) } \\
\hline & $\begin{array}{c}\text { \#Total } \\
\text { Via }\end{array}$ & $\begin{array}{c}\text { \#Alive } \\
\text { Via }\end{array}$ & $\begin{array}{l}\text { \#Ins. } \\
\text { Rvia }\end{array}$ & $\begin{array}{l}\text { Ins. } \\
\text { Rate }\end{array}$ & $\begin{array}{l}\text { \#On- } \\
\text { Track } \\
\text { Rvia }\end{array}$ & $\begin{array}{l}\text { On- } \\
\text { Track } \\
\text { Rate }\end{array}$ & $\begin{array}{l}\text { Time } \\
\text { (s) }\end{array}$ & $\begin{array}{l}\text { \#Ins. } \\
\text { Rvia }\end{array}$ & $\begin{array}{l}\text { Ins. } \\
\text { Rate }\end{array}$ & $\begin{array}{l}\text { \#On- } \\
\text { Track } \\
\text { Rvia }\end{array}$ & $\begin{array}{l}\text { On- } \\
\text { Track } \\
\text { Rate }\end{array}$ & $\begin{array}{c}\text { Time } \\
\text { (s) }\end{array}$ \\
\hline Mcc1 & 5501 & 5113 & 4908 & $96.0 \%$ & 3494 & $71.2 \%$ & 14.63 & 5012 & $98.0 \%$ & 3759 & $75.0 \%$ & 0.27 \\
\hline Mcc2 & 32148 & 29531 & 28650 & $97.0 \%$ & 21698 & $75.7 \%$ & 17.88 & 29101 & $98.5 \%$ & 21955 & $75.4 \%$ & 1.80 \\
\hline Struct & 7081 & 7039 & 7029 & $99.9 \%$ & 5552 & $79.0 \%$ & 27.46 & 7037 & $99.9 \%$ & 5912 & $84.0 \%$ & 0.24 \\
\hline Primary 1 & 5312 & 5249 & 5201 & $99.1 \%$ & 3853 & $74.1 \%$ & 37.84 & 5244 & $99.9 \%$ & 4296 & $81.9 \%$ & 0.16 \\
\hline Primary 2 & 22306 & 21871 & 21677 & $99.1 \%$ & 17370 & $80.1 \%$ & 32.84 & 21842 & $99.9 \%$ & 17803 & $81.5 \%$ & 0.76 \\
\hline S5378 & 6387 & 5942 & 5571 & $93.8 \%$ & 4181 & $75.0 \%$ & 1.56 & 5806 & $97.7 \%$ & 4468 & $77.0 \%$ & 0.20 \\
\hline S9234 & 5129 & 4789 & 4559 & $95.2 \%$ & 3228 & $70.8 \%$ & 32.24 & 4710 & $98.4 \%$ & 3724 & $79.1 \%$ & 0.15 \\
\hline S13207 & 13397 & 12610 & 11944 & $94.7 \%$ & 9258 & $77.5 \%$ & 12.45 & 12356 & $98.0 \%$ & 9843 & $79.7 \%$ & 0.44 \\
\hline S15850 & 16120 & 15041 & 14214 & $94.5 \%$ & 11072 & $77.9 \%$ & 26.21 & 14718 & $97.9 \%$ & 11559 & $78.5 \%$ & 0.53 \\
\hline S38417 & 39345 & 36861 & 35091 & $95.2 \%$ & 27621 & $78.7 \%$ & 8.43 & 36170 & $98.1 \%$ & 28741 & $79.5 \%$ & 1.41 \\
\hline S38584 & 52894 & 49561 & 46817 & $94.5 \%$ & 36314 & $77.6 \%$ & 28.84 & 48478 & $97.8 \%$ & 38208 & $78.8 \%$ & 2.02 \\
\hline \multicolumn{3}{|c|}{ Comp. } & 0.98 & $96.3 \%$ & - & $76.2 \%$ & 70.82 & 1.00 & $98.6 \%$ & - & $79.1 \%$ & 1.00 \\
\hline
\end{tabular}

TABLE VII

COMPARISON FOR DOUble-Via INSERTION With A COMMERCIAL TOOL ON THE MCNC BENCHMARKS

\begin{tabular}{|c|c|c||c|c|c||c|c|c|}
\hline & \multicolumn{2}{|c||}{ Via Info. } & \multicolumn{3}{c||}{ Commercial Tool } & \multicolumn{3}{c|}{ TDVI (Ours) } \\
\cline { 2 - 9 } Circuit & $\begin{array}{c}\text { \#Total } \\
\text { Single } \\
\text { Via }\end{array}$ & $\begin{array}{c}\text { \#Alive } \\
\text { Single } \\
\text { Via }\end{array}$ & $\begin{array}{c}\text { \#Ins. } \\
\text { Rvia }\end{array}$ & $\begin{array}{c}\text { Ins. } \\
\text { Rate }\end{array}$ & $\begin{array}{c}\text { Time } \\
\text { (s) }\end{array}$ & $\begin{array}{c}\text { \#Ins. } \\
\text { Rvia }\end{array}$ & $\begin{array}{c}\text { Ins. } \\
\text { Rate }\end{array}$ & $\begin{array}{c}\text { Time } \\
(\mathrm{s})\end{array}$ \\
\hline Mcc1 & 5948 & 5471 & 4224 & $77.2 \%$ & 10.0 & 5324 & $97.3 \%$ & 0.2 \\
Mcc2 & 34376 & 31212 & 26273 & $84.2 \%$ & 146.0 & 30684 & $98.3 \%$ & 1.0 \\
Struct & 7598 & 7543 & 5550 & $73.6 \%$ & 19.0 & 7445 & $99.9 \%$ & 0.2 \\
Primary1 & 5536 & 5468 & 4214 & $77.1 \%$ & 18.0 & 5417 & $99.1 \%$ & 0.2 \\
Primary2 & 23154 & 22663 & 17649 & $77.9 \%$ & 76.0 & 22486 & $99.2 \%$ & 0.8 \\
S5378 & 6739 & 6235 & 5697 & $91.4 \%$ & 12.0 & 6048 & $97.0 \%$ & 0.2 \\
S9234 & 5365 & 4991 & 4528 & $90.7 \%$ & 10.0 & 4854 & $97.3 \%$ & 0.2 \\
S13207 & 13972 & 13096 & 11930 & $91.1 \%$ & 48.0 & 12752 & $97.4 \%$ & 0.4 \\
S15850 & 16922 & 15693 & 14280 & $91.0 \%$ & 64.0 & 15256 & $97.2 \%$ & 0.5 \\
S38417 & 40942 & 38235 & 34625 & $90.6 \%$ & 318.0 & 37223 & $97.4 \%$ & 1.4 \\
S38584 & 55381 & 51602 & 46846 & $90.8 \%$ & 540.0 & 50133 & $97.2 \%$ & 2.0 \\
\hline \multicolumn{3}{|c|}{ Comp. } & 0.87 & $85.0 \%$ & 125.41 & 1.00 & $97.9 \%$ & 1.00 \\
\hline
\end{tabular}

for the routing results, "\#Ins. Rvia" shows the number of inserted double vias after the insertion process, "Ins. Rate" is equal to "\#Ins. Rvia" divided by "\#Alive Via," “\#On-Track Rvia" represents the number of on-track double vias, and "OnTrack Rate" is equal to "\#On-Track Rvia" divided by "\#Ins. Rvia." Compared with H2K_S, on average, TDVI obtains $299.3 \times$ runtime speedup and achieves a higher insertion rate at $98.6 \%$.

With the on-track/stacked redundant-via enhancement, we compared TDVI with H3K_S. Table VI shows the results. Compared with H3K_S, on average, TDVI obtains $70.8 \times$ runtime speedup and achieves a higher insertion rate at $98.6 \%$ with the on-track insertion rate at $79.1 \%$. The runtime improvement matches our expectation because the bipartite matching enjoys the polynomial-time complexity, whereas the MIS formulation is NP-complete. Thus, H2K_S and H3K_S need more running times for achieving high-quality solutions.

Tables VII and VIII show the double-via insertion results compared with the commercial tool for the MCNC and the Faraday benchmarks, respectively. For the MCNC benchmark, on average, TDVI can obtain $125.4 \times$ runtime speedup and achieve a higher insertion rate at $98.6 \%$; for the Faraday benchmark, TDVI can also obtain $6.1 \times$ runtime speedup and achieve a higher insertion rate at $99.80 \%$. 
TABLE VIII

COMPARISON FOR DOUble-VIA INSERTION WiTH A COMMERCIAL TOOL ON THE FARADAY BENCHMARKS

\begin{tabular}{|c|c|c|c|c|c|c|c|c|}
\hline \multirow[b]{2}{*}{ Circuit } & \multicolumn{2}{|c|}{ Via Info. } & \multicolumn{3}{|c|}{ Commercial Tool } & \multicolumn{3}{|c|}{ TDVI (Ours) } \\
\hline & $\begin{array}{c}\text { \#Total } \\
\text { Single Via }\end{array}$ & $\begin{array}{c}\text { \#Alive } \\
\text { Single Via }\end{array}$ & $\begin{array}{l}\text { \#Ins. } \\
\text { Rvia }\end{array}$ & $\begin{array}{l}\text { Ins. } \\
\text { Rate }\end{array}$ & $\begin{array}{l}\text { Time } \\
(\mathrm{sec})\end{array}$ & $\begin{array}{c}\text { \#Ins. } \\
\text { Rvia }\end{array}$ & $\begin{array}{l}\text { Ins. } \\
\text { Rate }\end{array}$ & $\begin{array}{l}\text { Time } \\
(\mathrm{sec})\end{array}$ \\
\hline DMA & 111047 & 71331 & 71192 & $99.81 \%$ & 28.00 & 71331 & $100.00 \%$ & 4.74 \\
\hline DSP1 & 225911 & 133449 & 132128 & $99.01 \%$ & 71.00 & 133198 & $99.81 \%$ & 11.34 \\
\hline DSP2 & 223684 & 131820 & 130719 & $99.16 \%$ & 66.00 & 131546 & $99.79 \%$ & 10.76 \\
\hline RISC1 & 342259 & 203055 & 199947 & $98.47 \%$ & 117.00 & 202487 & $99.72 \%$ & 19.51 \\
\hline RISC2 & 348005 & 207590 & 204262 & $98.97 \%$ & 119.00 & 206919 & $99.68 \%$ & 19.94 \\
\hline & Comp. & & 0.99 & $98.97 \%$ & 6.05 & 1.00 & $99.80 \%$ & 1.00 \\
\hline
\end{tabular}

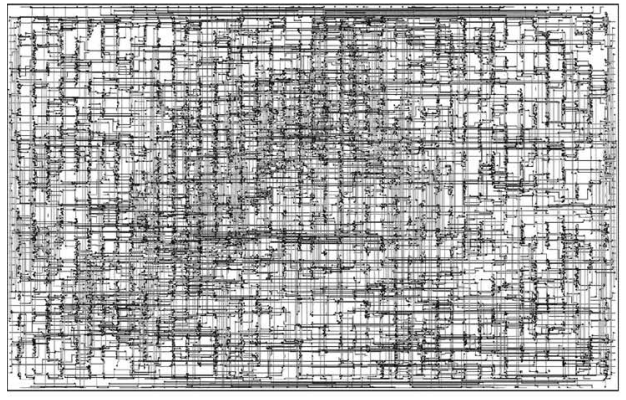

(a)

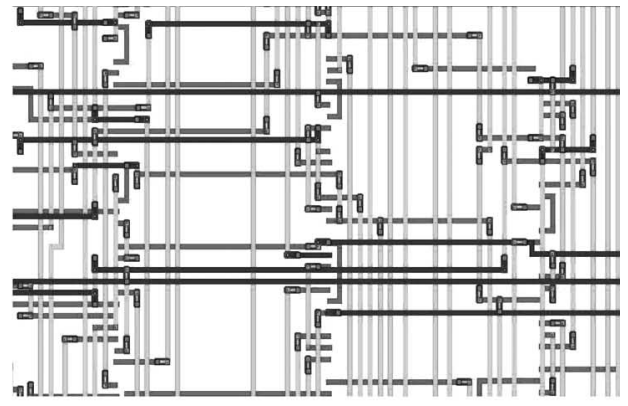

(b)

Fig. 15. Double-via insertion result for "S13207" obtained from TDVI. (a) Full-chip view (double vias are highlighted as black circles). (b) Local view.

Notice that, in Table $\mathrm{V}$, the insertion rates obtained by TDVI for all circuits, except "Mcc1" and "Mcc2," are optimal, because these designs contain only three routing layers. For "Mcc1," it is also reasonable that the number "\#Ins. Rvia" in Table VI is more than that in Table V, because the insertion process is not optimal for designs with more than three routing layers. Fig. 15(a) and (b) shows the PDVI result for "S13207" with full-chip and local views, respectively.

\section{CONCLUSION}

We have presented a new two-pass bottom-up full-chip gridless router, named TBR, considering double-via insertion for yield enhancement. We have also proposed an optimal polynomial-time PDVI algorithm for grid-based routing with up to three routing layers and the stacked-via structure and have extended the algorithm to handle the general problem. Experimental results have shown the effectiveness and efficiency of the proposed methods.

Future work lies in performing wire perturbation or spreading and/or extending wires to allocate additional space for each dead via to further improve the insertion rate. To add more redundant vias for each alive via, furthermore, we may iteratively perform our TDVI algorithm, i.e., we first insert the first redundant via for each alive via, then find an additional redundant-via candidate in the updated layout and insert the second redundant via, and so on.

\section{ACKNOWLEDGMENT}

The authors would like to thank the Editor, the Associate Editor, and the reviewers for the very prompt handling and the very constructive comments.

\section{REFERENCES}

[1] S. N. Adya, S. Chaturvedi, J. A. Roy, D. Papa, and I. L. Markov, "Unification of partitioning, floorplanning and placement," in Proc. IEEE/ACM Int. Conf. Comput.-Aided Des., Nov. 2004, pp. 550-557.

[2] C. Albrecht, "Global routing by new approximation algorithms for multicommodity flow," IEEE Trans. Comput.-Aided Design Integr. Circuits Syst., vol. 20, no. 5, pp. 622-632, May 2001.

[3] G. A. Allan, "Targeted layout modifications for semiconductor yield/reliability enhancement," IEEE Trans. Semicond. Manuf., vol. 17, no. 4, pp. 573-581, Nov. 2004.

[4] U. Brenner and A. Rohe, "An effective congestion-driven placement framework," IEEE Trans. Comput.-Aided Design Integr. Circuits Syst., vol. 22, no. 4, pp. 387-394, Apr. 2003.

[5] QUALEX package. [Online]. Available: http://www.busygin.dp.uanpc. $\mathrm{html}$

[6] Y.-W. Chang and S.-P. Lin, "MR: A new framework for multilevel fullchip routing," IEEE Trans. Comput.-Aided Design Integr. Circuits Syst., vol. 23, no. 5, pp. 793-800, May 2004.

[7] H.-Y. Chen, M.-F. Chiang, Y.-W. Chang, L. Chen, and B. Han, "Novel full-chip gridless routing considering double-via insertion," in Proc. ACM/IEEE Des. Autom. Conf., Jun. 2006, pp. 755-760.

[8] T.-C. Chen and Y.-W. Chang, "Multilevel gridless routing considering optical proximity correction," in Proc. IEEE/ACM Asia South Pacific Des. Autom. Conf., Jan. 2005, pp. 1160-1163.

[9] T.-C. Chen, Y.-W. Chang, and S.-C. Lin, "A novel framework for multilevel full-chip gridless routing," in Proc. IEEE/ACM Asia South Pacific Des. Autom. Conf., Jan. 2006, pp. 636-641.

[10] J. Cong, J. Fang, M. Xie, and Y. Zhang, "MARS-A multilevel fullchip gridless routing system," IEEE Trans. Comput.-Aided Design Integr. Circuits Syst., vol. 24, no. 3, pp. 382-394, Mar. 2005.

[11] J. Cong, J. Fang, and Y. Zhang, "Multilevel approach to full-chip gridless routing," in Proc. IEEE/ACM Int. Conf. Comput.-Aided Des., Nov. 2001, pp. 396-403.

[12] J. Heisterman and T. Lengauer, "The efficient solutions of integer programs for hierarchical global routing," IEEE Trans. Comput.-Aided Design Integr. Circuits Syst., vol. 10, no. 6, pp. 748-753, Jun. 1991.

[13] T.-Y. Ho, Y.-W. Chang, S.-J. Chen, and D.-T. Lee, "Crosstalk- and performance-driven multilevel full-chip routing," IEEE Trans. Comput.Aided Design Integr. Circuits Syst., vol. 24, no. 6, pp. 869-878, Jun. 2005.

[14] Y.-L. Hsieh and T.-M. Hsieh, "A new effective congestion model in floorplan design," in Proc. IEEE/ACM Des., Autom. Test Eur. Conf., Mar. 2004, pp. 1204-1209.

[15] R. M. Karp, "Reducibility among combinatorial problems," in Proc. Symp. Complexity Computer Comput., 1972, pp. 85-103. 
[16] R. Kastner, E. Bozorgzadeh, and M. Sarrafzadeh, "Pattern routing: Use and theory for increasing predictability and avoiding coupling," IEEE Trans. Comput.-Aided Design Integr. Circuits Syst., vol. 21, no. 7, pp. 777-790, Nov. 2002.

[17] C. Y. Lee, "An algorithm for path connection and its application," IRE Trans. Electron. Comput., vol. 10, pp. 346-365, 1961.

[18] K.-Y. Lee and T.-C. Wang, "Post-routing redundant via insertion for yield/reliability improvement," in Proc. IEEE/ACM Asia South Pacific Des. Autom. Conf., Jan. 2006, pp. 303-308.

[19] Y.-L. Lin, Y.-C. Hsu, and F.-S. Tsai, "Hybrid routing," IEEE Trans. Comput.-Aided Design Integr. Circuits Syst., vol. 9, no. 2, pp. 151-157, Feb. 1990.

[20] J. Lou, S. Thakur, S. Krishnamoorthy, and H. S. Sheng, "Estimating routing congestion using probabilistic analysis," IEEE Trans. Comput.Aided Design Integr. Circuits Syst., vol. 21, no. 1, pp. 32-41, Jan. 2002.

[21] M. Marek-Sadowska, "Global router for gate array," in Proc. IEEE Int. Conf. Comput. Des., Oct. 1984, pp. 332-337.

[22] Taiwan Semiconductor Manufacturing Company (TSMC). Reference Flow 5.0 and Reference Flow 6.0.

[23] D. B. West, Introduction to Graph Theory, 2nd ed. Englewood Cliffs, NJ: Prentice-Hall, 2001.

[24] J. G. Xi, Improving Yield in RTL-to-GDSII Flows. Manhasset, NY: EE Times, Jul. 11, 2005.

[25] J. Xiong and L. He, "Probabilistic congestion model considering shielding for crosstalk reduction," in Proc. IEEE/ACM Asia South Pacific Des. Autom. Conf., Jan. 2005, pp. 739-742.

[26] G. Xu, L.-D. Huang, D. Z. Pan, and M. D. Wong, "Redundant-via enhanced maze routing for yield improvement," in Proc. IEEE/ACM Asia South Pacific Des. Automa. Conf., Jan. 2005, pp. 1148-1151.

[27] H. Yao, Y. Cai, X. Hong, and Q. Zhou, "Improved multilevel routing with redundant via placement for yield and reliability," in Proc. ACM Great Lakes Symp. VLSI, 2005, pp. 143-146.

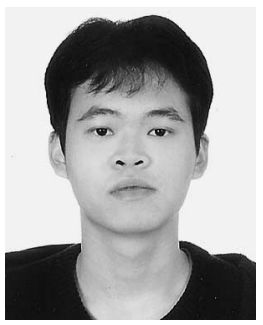

Huang-Yu Chen (S'05) received the B.S. degree in electrical engineering from National Tsing Hua University, Hsinchu, Taiwan, R.O.C., in 2004. He is currently working toward the $\mathrm{Ph} . \mathrm{D}$. degree in the Graduate Institute of Electronics Engineering, National Taiwan University, Taipei, Taiwan. His research interests include physical design for VLSI circuits and manufacturability-driven large-scale routing.

Mr. Chen was the recipient of the 2006 Outstanding Research Award from the National Taiwan University and the Best Paper Nomination from ICCAD 2007.

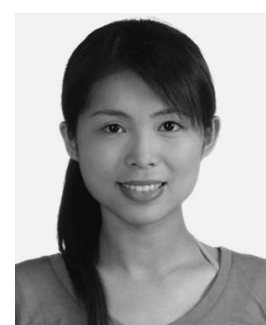

Mei-Fang Chiang (S'05) received the B.S. degree in computer science from National Tsing Hua University, Hsinchu, Taiwan, R.O.C., in 2004 and the M.S. degree in electrical engineering from National Taiwan University, Taipei, Taiwan, in 2006. She is currently working toward the Ph.D. degree in the Graduate School of Information, Production and Systems, Waseda University, Kitakyushu, Japan.

Her current research interests include the design for manufacturability.

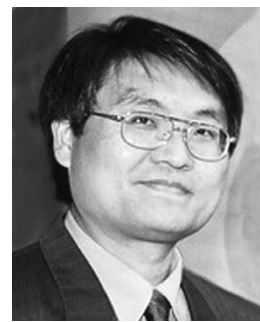

Yao-Wen Chang (S'94-M'96) received the B.S degree from National Taiwan University, Taipei, Taiwan, R.O.C., in 1988, and the M.S. and Ph.D. degrees from the University of Texas, Austin, in 1993 and 1996, respectively, all in computer science.

$\mathrm{He}$ is currently a Professor with the Graduate Institute of Electronics Engineering and Department of Electrical Engineering, National Taiwan University. His current research interests include physical design for VLSI circuits, design for manufacturability, and design automation for biochips.

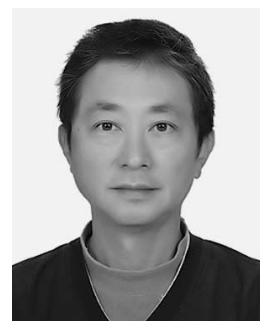

Lumdo Chen received the B.S. and M.S. degrees in electrical engineering from National Chiao Tung University, Hsinchu, Taiwan R.O.C., in 1982 and 1986, respectively.

$\mathrm{He}$ has been with United Microelectronic Corporation (UMC), Hsinchu, for 21 years. He was a Microcontroller Design Manager in the late 1980s and a Corporate CAD Manager in the 1990s. He is currently the Chairman of the EDA committee with UMC.

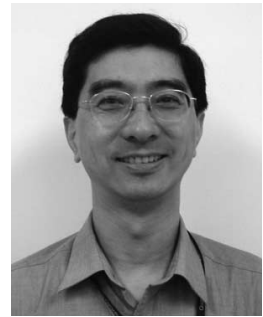

Brian Han received the B.S. degrees in electrical engineering from National Tsing Hua University, Hsinchu, Taiwan, R.O.C., in 1985, and the M.S degree in electrical engineering from Polytechnic University, Brooklyn, NY, in 1989.

He has been with several companies both in the USA and Taiwan for 17 years. The major fields he works on are CAD tool development and ASIC design. He is currently the Department Manager of the CAD Group, United Microelectronic Corporaology and design automation. 